\title{
A solution of a problem of Sophus Lie: Normal forms of 2-dim metrics admitting two projective vector fields.
}

\author{
Robert L. Bryant, Gianni Manno†, Vladimir S. Matveev ${ }^{\ddagger}$
}

\begin{abstract}
We give a complete list of normal forms for the 2-dimensional metrics that admit a transitive Lie pseudogroup of geodesic-preserving transformations and we show that these normal forms are mutually non-isometric. This solves a problem posed by Sophus Lie.
\end{abstract}

\section{Introduction}

\subsection{Main definition and result}

Let $g$ be a Riemannian or pseudo-Riemannian metric on a 2-dimensional surface $D$. A diffeomorphism $\phi: U_{1} \rightarrow U_{2}$ between two open subsets of $D$ is said to be projective with respect to $g$ if it takes the unparametrized geodesics of $g$ in $U_{1}$ to the unparametrized geodesics of $g$ in $U_{2}$.

Lie showed that what is (nowadays) called the pseudo-group $P(g)$ of projective transformations of $g$ is a Lie pseudo-group. A vector field $v$ on $D$ is said to be projective with respect to $g$, if its (locally defined) flow belongs to $P(g)$. As Lie showed, the set of vector fields projective with respect to a given $g$ forms a Lie algebra. We will denote this Lie algebra by $\mathfrak{p}(g)$.

Clearly, any Killing vector field for $g$ is projective with respect to $g$. Metrics of constant curvature give examples of metrics admitting projective vector fields that are not Killing. It has been known since the time of Lagrange and Beltrami [3, 14 that a metric $g$ of constant curvature on a simply connected domain $D$ has $\operatorname{dim}(\mathfrak{p}(g))=8$. (In fact, in this case, $\mathfrak{p}(g) \simeq \mathfrak{s l}(3, \mathbb{R})$ as Lie algebras.)

The following problem was posed by Sophus Lif1 in 1882:

Problem (Lie). Find all metrics $g$ such that $\operatorname{dim} \mathfrak{p}(g) \geq 2$.

\footnotetext{
*Duke University Mathematics, PO Box 90320, Durham, NC 27708

${ }^{\dagger}$ Department of Mathematics, via per Arnesano, 73100 Lecce Italy

${ }^{\ddagger}$ Institute of Mathematics, FSU Jena, 07737 Jena Germany, matveev@minet.uni-jena.de

1 German original from [15], Abschn. I, Nr. 4, Problem II: Man soll die Form des Bogenelementes einer jeden Fläche bestimmen, deren geodätische Kurven mehrere infinitesimale Transformationen gestatten.
} 
The following theorem, which is the main result of our paper, essentially solves this problem.

Theorem 1. Suppose that a metric $g$ on $D^{2}$ possesses two projective vector fields that are linearly independent at some $p \in D^{2}$. Then either $g$ has constant curvature on some neighborhood of $p$ or else, on some neighborhood of $p$, there exist coordinates $x, y$ in which the metric $g$ takes one of the following forms:

1. Metrics with $\operatorname{dim} \mathfrak{p}(g)=2$.

(a) $\varepsilon_{1} e^{(b+2) x} d x^{2}+\varepsilon_{2} e^{b x} d y^{2}$, where $b \in \mathbb{R} \backslash\{-2,0,1\}$ and $\varepsilon_{i} \in\{-1,1\}$ are constants,

(b) $a\left(\frac{e^{(b+2) x} d x^{2}}{\left(e^{b x}+\varepsilon_{2}\right)^{2}}+\varepsilon_{1} \frac{e^{b x} d y^{2}}{e^{b x}+\varepsilon_{2}}\right)$, where $a \in \mathbb{R} \backslash\{0\}, b \in \mathbb{R} \backslash\{-2,0,1\}$, and $\varepsilon_{i} \in\{-1,1\}$ are constants, and

(c) $a\left(\frac{e^{2 x} d x^{2}}{x^{2}}+\varepsilon \frac{d y^{2}}{x}\right)$, where $a \in \mathbb{R} \backslash\{0\}$, and $\varepsilon \in\{1,-1\}$ are constants.

2. Metrics with $\operatorname{dim} \mathfrak{p}(g)=3$.

(a) $\varepsilon_{1} e^{3 x} d x^{2}+\varepsilon_{2} e^{x} d y^{2}$, where $\varepsilon_{i} \in\{-1,1\}$ are constants,

(b) $a\left(\frac{e^{3 x} d x^{2}}{\left(e^{x}+\varepsilon_{2}\right)^{2}}+\varepsilon_{1} \frac{e^{x} d y^{2}}{\left(e^{x}+\varepsilon_{2}\right)}\right)$, where $a \in \mathbb{R} \backslash\{0\}, \varepsilon_{i} \in\{-1,1\}$ are constants, and

(c) $a\left(\frac{d x^{2}}{\left(c x+2 x^{2}+\varepsilon_{2}\right)^{2} x}+\varepsilon_{1} \frac{x d y^{2}}{\left(c x+2 x^{2}+\varepsilon_{2}\right)}\right)$, where $a>0, \varepsilon_{i} \in\{-1,1\}, c \in \mathbb{R}$ are constants.

No two distinct metrics from this list are isometric.

Strictly speaking, what Theorem 1 gives is a list of local normal forms for metrics $g$ whose projective pseudo-group $P(g)$ is locally transitive. Naturally, for such metrics, one has $\operatorname{dim} \mathfrak{p}(g) \geq 2$. In Sections 2.2.2 and 2.2.3] it will be shown that, for a metric $g$ of nonconstant curvature on a surface $D$, any two-dimensional subalgebra $\mathfrak{s} \subset \mathfrak{p}(g)$ acts locally transitively on a dense open subset of $D$. (One can further show that this open set has full measure.) Thus, if $\operatorname{dim} \mathfrak{p}(g) \geq 2$, then either $g$ has constant curvature or else, outside a closed set with no interior $2 g$ is locally isometric to one of the metrics given in Theorem 1 .

Remark 1. The higher dimensional analog of Lie's question is easier, possibly because the systems of PDE that appear have a higher degree of overdeterminacy. It was treated with success by Solodovnikov [33, 34, 35. The global (i.e., when the manifold and the projective vector fields are complete) version of Lie's question was explicitly asked by Schouten [32]; for the Riemannian case, it was solved in [22, 23, 24, 25].

\footnotetext{
${ }^{2}$ Examples show that this closed set can be nonempty.
} 


\subsection{Killing vector fields for metrics with $\operatorname{dim} \mathfrak{p}(g) \geq 2$ and results of A.V. Aminova}

It appears to be commonly believed that A.V. Aminova solved Lie's problem in 1, 2. Indeed, she claimed

Theorem 2. If a metric $g$ satisfies $\operatorname{dim} \mathfrak{p}(g) \geq 2$, then it admits a Killing vector field.

She then used this result to classify the metrics $g$ with $\operatorname{dim}(\mathfrak{p}(g)) \geq 2$.

Unfortunately, Aminova's proof of Theorem 2 has a serious gap. Namely, she assumed (see [1, pp. 3,6] or [2, pp. 414,424]) that Koenigs [12] had proved that if the geodesic flow of a metric admits three linearly independent quadratic integrals 3 , then the metric admits a nontrivial Killing vector field.

However, a careful reading of Koenigs' note [12] shows that he had instead proved that if the geodesic flow of a metric admits four linearly independent quadratic integrals, then the metric admits a nontrivial Killing vector field.

Indeed, Koenigs' examples already show that the existence of three linearly independent quadratic integrals does not imply the existence of a nontrivial Killing vector field.

Example 1 (12]). Consider the metric $g$ given by $\left(4 x^{2}+y^{2}+1\right)\left(d x^{2}+d y^{2}\right)$. The geodesic flow of this metric has three linearly independent quadratic integrals. They are

$$
\begin{aligned}
& F_{0}=\left(4 x^{2}+y^{2}+1\right)\left(d x^{2}+d y^{2}\right), \\
& F_{1}=\left(4 x^{2}+y^{2}+1\right)\left(y^{2} d x^{2}-\left(4 x^{2}+1\right) d y^{2}\right), \\
& F_{2}=\left(4 x^{2}+y^{2}+1\right)\left(x y^{2}\left(d x^{2}+d y^{2}\right)+\left(4 x^{2}+y^{2}+1\right) d y(x d y-y d x)\right) .
\end{aligned}
$$

However this metric admits no nontrivial Killing vector field. Indeed, the scalar curvature $R$ of this metric and its $g$-Laplacian $\Delta_{g} R$ are independent functions, which is impossible for metrics admitting nontrivial Killing vector fields.

On the other hand, all the normal forms listed in Theorem 1 admit $\frac{\partial}{\partial y}$ as a Killing vector field. Thus, Theorem 1 implies Theorem 2 (in the proof of Corollary 3 we will show, that if a projective vector field is a Killing vector field on an open subset, then it is a Killing vector field on the whole connected manifold). Consequently, Aminova's description of the metrics $g$ with $\operatorname{dim} \mathfrak{p}(g) \geq 2$ is correct and can be obtained from ours by coordinate changes. However, we prefer our description because it is simpler. For example, all the metrics in our list are given by elementary functions, while some of Aminova's metrics include functions implicitly given as a solution of a certain differential equation.

Finally, we would like to remark that we have not found a way to prove Theorem 2 directly, i.e., without constructing the list of the metrics first.

\footnotetext{
${ }^{3}$ For a discussion of the notion of 'quadratic integrals' of a geodesic flow and their independence, see Section 2.2 .4
} 


\section{Acknowledgements}

The first author acknowledges financial support from the National Science Foundation via DMS-0604195 and from Duke University. The second author would like to express his gratitude to Katholieke Universiteit Leuven for hospitality, and to R. Vitolo for useful discussions. The third author would like to thank V. Bangert, A. Bolsinov, A. Cap, B. Doubrov, M. Eastwood, O. Kowalski, B. Kruglikov, and V. Shevchishin for useful discussions, Deutsche Forschungsgemeinschaft (Priority Program 1154 - Global Differential Geometry), Ministerium für Wissenschaft, Forschung und Kunst Baden-Württemberg (Eliteförderprogramm Postdocs 2003), KU Leuven, and FSU Jena for partial financial support, and University of Lecce and Duke University for hospitality.

\section{Proof}

\subsection{Outline of the proof}

In Section 2.2 we recall some classical results of Lie, Liouville, Tresse, Cartan, and Koenigs. In Section 2.3 we use these results to describe all projective connections admitting two projective vector fields. In Sections 2.4 and 2.5 we determine which of these connections come from (pseudo)Riemannian metrics. In order to do this, given a projective connection, we first find a system of PDE such that the existence of nontrivial solutions of this system implies the existence of metrics with this projective connection (see Lemma 5 in Section 2.4). This equation (10) in Section 2.4 ) is seen to be linear in the components of the matrix $g / \operatorname{det}(g)^{2 / 3}$.

In Section 2.5 we use the linearity of the equations and the good normal form for the projective connection from Section 2.3 to reduce this system of partial differential equations to a system of ordinary differential equations under the additional assumption that the metric is not superintegrable 5 . Then we solve this system of ODE and obtain the list of the metrics. In Section 2.6.3 we describe all metrics $g$ with nonconstant curvature and $\operatorname{dim} \mathfrak{p}(g) \geq 3$ (which turn out to be preciesly the superintegrable ones, see Lemma 2 and Corollary 3 ). Finally, in Section 2.7 we explain why the metrics from Theorem 1 are mutually nonisometric.

\footnotetext{
${ }^{4}$ This linearity observation is a coordinate-free version of an observation that Darboux attributed to Dini. See Darboux [6 $\$ \S 600-608]$. However, the general result was already known to R. Liouville in 1887 [18.

${ }^{5}$ We recall the definition of superintegrable metrics in Section 2.2.4 We also prove that superintegrable metrics are those admitting precisely three linearly independent projective vector fields.
} 


\subsection{Classical results of Beltrami, Lie, Liouville, Koenigs, Cartan, and Tresse}

\subsubsection{Projective connections}

A second order ordinary differential equation of the form

$$
y^{\prime \prime}=K^{0}(x, y)+K^{1}(x, y) y^{\prime}+K^{2}(x, y)\left(y^{\prime}\right)^{2}+K^{3}(x, y)\left(y^{\prime}\right)^{3},
$$

where the functions $K^{i}$ are defined on some connected domain $D$ in the $x y$ plane, is classically referred to as a projective connection 6

For any symmetric affine connection on the domain $D^{2}$ with coordinates $x, y$, say, $\Gamma(x, y)=\left(\Gamma_{j k}^{i}(x, y)\right)=\left(\Gamma_{k j}^{i}(x, y)\right)$, the projective connection associated to $\Gamma$ is defined to be

$$
y^{\prime \prime}=-\Gamma_{11}^{2}+\left(\Gamma_{11}^{1}-2 \Gamma_{12}^{2}\right) y^{\prime}-\left(\Gamma_{22}^{2}-2 \Gamma_{12}^{1}\right)\left(y^{\prime}\right)^{2}+\Gamma_{22}^{1}\left(y^{\prime}\right)^{3} .
$$

It has been known since the time of Beltrami [3], that the solutions of this ODE and the geodesics of the connection $\Gamma$ are closely related: Namely for every solution $y(x)$ of (2) the curve $(x, y(x))$ is, up to reparametrization, a geodesic of $\Gamma$.

It is well-known (and easy to verify directly) that, under any coordinate change of the form $(x, y)=(F(\bar{x}, \bar{y}), G(\bar{x}, \bar{y}))$, the projective connection (11) is transformed into another projective connection, now expressed in terms of $(\bar{x}, \bar{y})$.

A vector field 7

$$
Z=Z^{1}(x, y) \frac{\partial}{\partial x}+Z^{2}(x, y) \frac{\partial}{\partial y}
$$

on $D$ is an infinitesimal symmetry of the projective connection (11) if its (local) flow preserves (11). It is known [15, 31, that a vector field $Z$ as above is an infinitesimal symmetry of (11) if and only if it satisfies the PDE system

$$
\left.\begin{array}{r}
Z_{x x}^{2}-2 K^{0} Z_{x}^{1}-K^{1} Z_{x}^{2}+K^{0} Z_{y}^{2}-K_{x}^{0} Z^{1}-K_{y}^{0} Z^{2}=0 \\
-Z_{x x}^{1}+2 Z_{x y}^{2}-K^{1} Z_{x}^{1}-3 K^{0} Z_{y}^{1}-2 K^{2} Z_{x}^{2}-K_{x}^{1} Z^{1}-K_{y}^{1} Z^{2}=0 \\
-2 Z_{x y}^{1}+Z_{y y}^{2}-2 K^{1} Z_{y}^{1}-3 K^{3} Z_{x}^{2}-K^{2} Z_{y}^{2}-K_{x}^{2} Z^{1}-K_{y}^{2} Z^{2}=0 \\
-Z_{y y}^{1}+K^{3} Z_{x}^{1}-K^{2} Z_{y}^{1}-2 K^{3} Z_{y}^{2}-K_{x}^{3} Z^{1}-K_{y}^{3} Z^{2}=0
\end{array}\right\}
$$

The set of vector fields in $D$ whose flows preserve the projective connection (11) form a Lie algebra, which will be denoted $\mathfrak{p}(D$, (10), or, more simply, $\mathfrak{p}($ (1) $)$ when the domain $D$ is clear from context. We will also use the notation $\mathfrak{p}(\Gamma)$ for the equation (2), and for a metric $g$, we will use the notation $\mathfrak{p}(g)$ to denote infinitesimal symmetries of the the projective connection associated to the Levi-Civita connection of $g$.

\footnotetext{
${ }^{6}$ For the relation of this classical notion with the modern formulation of projective connections due to Cartan, see [5].

${ }^{7}$ When the $x y$-coordinates are clear from context, we will also use the more compact notation $Z=\left(Z^{1}, Z^{2}\right)$ for this vector field.
} 
For use below, we want to point out the following consequence of (3): Differentiating the equations (3) with respect to $x$ and $y$, one obtains a system of 8 third order equations for the $Z^{i}$ that can be solved for all of the third order derivatives of the $Z^{i}$ as linear expressions in their lower order derivatives.

In particular, consider the $\mathbb{R}^{8}$-valued function

$$
\hat{Z}=\left(Z^{1}, Z^{2}, Z_{x}^{1}, Z_{x}^{2}, Z_{y}^{1}, Z_{y}^{2}, Z_{x y}^{1}, Z_{x y}^{2}\right) .
$$

The system (3) and its derivatives can be written in the vectorial form

$$
\mathrm{d} \hat{Z}=\hat{Z}(X \mathrm{~d} x+Y \mathrm{~d} y) .
$$

where $X=X(x, y)$ and $Y=Y(x, y)$ are certain 8-by-8 matrices whose entries are constructed from the functions $K^{i}$ and their first two derivatives. One can thus regard the $\mathfrak{g l}(8, \mathbb{R})$-valued 1-form $\psi=(X \mathrm{~d} x+Y \mathrm{~d} y)$ as defining a linear connection on the bundle $D \times \mathbb{R}^{8}$, one whose parallel sections $\hat{Z}$ on an open set $U \subset D$ correspond to the elements of $\mathfrak{p}(U,(\mathbb{1}))$. This interpretation of (3) has some useful consequences 8

First, if $U \subset D$ is connected, then, for any point $p \in U$, the evaluation mapping

$$
\mathrm{ev}_{p}(Z)=\left(Z^{1}(p), Z^{2}(p), Z_{x}^{1}(p), Z_{x}^{2}(p), Z_{y}^{1}(p), Z_{y}^{2}(p), Z_{x y}^{1}(p), Z_{x y}^{2}(p)\right)
$$

defines a linear injection $\mathrm{ev}_{p}: \mathfrak{p}(U,(\mathbb{1})) \rightarrow \mathbb{R}^{8}$. In particular, if any $\left.Z \in \mathfrak{p}(\mathbb{1})\right)$ vanishes to order 3 at any point of $D$ (assumed connected), then $Z$ vanishes identically.

Second, for any $p \in D$, one can define its local infinitesimal symmetry algebra $\mathfrak{p}(p$, (1) $)$ to be the inverse limit of the symmetry algebras $\mathfrak{p}(U$, (1) $)$ as $U$ ranges over the open neighborhoods of $p$ in $D$. The above formulation then implies that $\mathfrak{p}(p$, (1) $)=\mathfrak{p}(U$, (1) $)$ for some connected open neighborhood $U$ of $p$ in $D$.

Third, differentiating $\mathrm{d} \hat{Z}=\hat{Z} \psi$ yields $0=\mathrm{d}(\mathrm{d} \hat{Z})=\hat{Z}(\mathrm{~d} \psi+\psi \wedge \psi)$, so that one gets the relation $\hat{Z} L=0$, where

$$
\mathrm{d} \psi+\psi \wedge \psi=\left(Y_{x}-X_{y}+[X, Y]\right) \mathrm{d} x \wedge \mathrm{d} y=L(x, y) \mathrm{d} x \wedge \mathrm{d} y
$$

is the curvature form of the connection $\psi$. Thus, if $L(p)$ is nonzero, one gets that $\operatorname{dim} \mathfrak{p}(p, \mathbb{1})<8$. Also, when $L$ is nonzero, one gets more relations on $\hat{Z}$ by differentiating $0=\hat{Z} L$, which yields $0=\hat{Z}(\mathrm{~d} L+\psi L)$. On the other hand, when $L$ vanishes identically on a simply-connected open $p$-neighborhood $U$, the flatness of the connection $\psi$ implies that $\operatorname{dim} \mathfrak{p}(U, \mathbb{1}))=8$.

\footnotetext{
${ }^{8}$ For those interested in an invariant formulation, we offer the following description: The linear differential equations (3) define in $J^{2}(D, T D)$, the bundle of 2-jets of vector fields on $D$, a vector subbundle $P \subset J^{2}(D, T D)$ of rank 8. The contact plane field on $J^{2}(D, T D)$ (i.e., the plane field of codimension 6 to which all of the holonomic sections of $J^{2}(D, T D)$ are tangent) produces a horizontal 2-plane field $H \subset T P$ that defines a linear connection on the bundle $P \rightarrow D$. The 2 -jets of projective vector fields on $D$ are then sections of $P$ that are parallel with respect to this connection.
} 


\subsubsection{Lie algebras of projective vector fields}

Lie [15] classified the possible local infinitesimal symmetry algebras of projective vector fields of projective connections. He proved 9 that such a local Lie algebra is isomorphic to one of the following

$$
\begin{array}{llll}
\text { 1. }\{0\}, \quad \text { 2. } \mathbb{R}, & \text { 3. } \mathfrak{s}, \quad \text { 4. } \mathfrak{s l}(2, \mathbb{R}), \quad 5 . \mathfrak{s l}(3, \mathbb{R}) .
\end{array}
$$

where $\mathfrak{s}$ is the non-commutative Lie algebra of dimension 2 (i.e., the algebra spanned by two elements $X$ and $Y$ that satisfy $[X, Y]=X$ ). Since we will be considering the cases when a metric has at least two linearly independent projective vector fields, the first two algebras will not arise.

Both of the algebras $\mathfrak{s l}(2, \mathbb{R})$ and $\mathfrak{s l}(3, \mathbb{R})$ contain $\mathfrak{s}$ as a subalgebra. Thus, by Lie's classification, if a connection $\Gamma$ satisfies $\operatorname{dim} \mathfrak{p}(\Gamma) \geq 2$, it admits two projective vector fields $X \not \equiv 0$ and $Y$ satisfying the relation $[X, Y]=X$.

Lie [17] also investigated the possible realizations of $\mathfrak{s}$ as an algebra of vector fields on $\mathbb{R}^{2}$. He showed that, for two vector fields $X, Y$ on $\mathbb{R}^{2}$ satisfying the relation $[X, Y]=X$, almost every point $p \in \mathbb{R}^{2}$ has a neighborhood on which there are coordinates $x, y$ in which

$$
\begin{aligned}
& \text { transitive case } X=\frac{\partial}{\partial y}, Y=\frac{\partial}{\partial x}+y \frac{\partial}{\partial y}, \\
& \text { nontransitive case } X=e^{y} \frac{\partial}{\partial y}, Y=-\frac{\partial}{\partial y}, \text { or } \\
& \text { trivial case } X \equiv 0 .
\end{aligned}
$$

Note that there exist examples of smooth vector fields $X \not \equiv 0$ and $Y$ on the plane that satisfy the relation $[X, Y]=X$ and are such that they are as in the transitive case in some open set and as in the trivial case in another open set.

Example 2. Let the function $f: \mathbb{R} \rightarrow \mathbb{R}$ be given by

$$
f(y)= \begin{cases}e^{-\frac{1}{2 y^{2}}} & \text { for } y>0 \\ 0 & \text { for } y \leq 0 .\end{cases}
$$

Then the vector fields $X=f(y) \frac{\partial}{\partial x}$ and $Y=-y^{3} \frac{\partial}{\partial y}$, satisfy $[X, Y]=X$ and are as in the transitive case for $y>0$ but are as in the trivial case for $y<0$.

Moreover, the vector fields $X=\frac{\partial}{\partial x}$ and $Y=x \frac{\partial}{\partial x}+f(y) \frac{\partial}{\partial y}$ also satisfy $[X, Y]=X$ and fall into the transitive case for $y>0$ but into the nontransitive case for $y<0$.

From the results of Section 2.2.1 it follows, that this phenomena cannot happen, if the vector fields are infinitesimal symmetries of a projective connection (on a connected $D$ ). Indeed, if $X \in \mathfrak{p}($ (1) $)$ vanishes at every point of an open subset $U$, then for every $p \in U$ we evidently have $\operatorname{ev}_{p}(X)=0$ implying $X \equiv 0$.

Thus, if two infinitesimal symmetries $X, Y$ of a projective connection on a connected surface satisfy $[X, Y]=X$ and $X \not \equiv 0$, then, in a neighborhood of almost every point, they are as in the transitive case, or as in the nontransitive case.

${ }^{9}$ Modern version of Lie's proof can be found, for example, in [9, 27]. 


\subsubsection{Invariants that decide whether a projective connection corre- sponds to the metric of constant curvature.}

We say that a projective connection on $D^{2}$ is flat, if every point of $D^{2}$ has local coordinates $x, y$ such that the projective connection has the form $y^{\prime \prime}=0$ (i.e., if the geodesics of the projective connection can be mapped into straight lines). Lie [15] showed that the projective connection of a metric is flat if and only if the metric has constant curvature, and that the (local) Lie algebra $\mathfrak{p}(g)$ of a metric $g$ of constant curvature is $\mathfrak{s l}(3, \mathbb{R})$. (Of course, this follows immediately from Beltrami's earlier result that the geodesics of a metric can be mapped to straight lines in the plane if and only if the metric has constant curvature.)

There is a simple test for when the projective connection (1) is flat. Consider the following two functions (sometimes improperly called Cartan invariants although they were already known to Liouville [18] in 1889):

$$
\begin{array}{r}
L_{1}=2 K_{x y}^{1}-K_{x x}^{2}-3 K_{y y}^{0}-6 K^{0} K_{x}^{3}-3 K^{3} K_{x}^{0} \\
+3 K^{0} K_{y}^{2}+3 K^{2} K_{y}^{0}+K^{1} K_{x}^{2}-2 K^{1} K_{y}^{1} \\
\begin{aligned}
L_{2}=2 & K_{x y}^{2}-K_{y y}^{1}-3 K_{x x}^{3}+6 K^{3} K_{y}^{0}+3 K^{0} K_{y}^{3} \\
& -3 K^{3} K_{x}^{1}-3 K^{1} K_{x}^{3}-K^{2} K_{y}^{1}+2 K^{2} K_{x}^{2}
\end{aligned}
\end{array}
$$

Liouville [18] proved that the expression

$$
\lambda=\left(L_{1} \mathrm{~d} x+L_{2} \mathrm{~d} y\right) \otimes(\mathrm{d} x \wedge \mathrm{d} y)
$$

is a differential invariant 10 (w.r.t. coordinate changes) of the projective connection (11). Moreover, Liouville [18, Tresse [31, and Cartan [5] each gave independent proofs that a projective connection is flat on an open set $U$ if and only $\lambda$ vanishes there.

Remark 2. A calculation shows that the equation $\hat{Z} L=0$ from Section 2.2.1 is just the condition that the Lie derivative of $\lambda$ with respect to $Z$ be zero, see for example [27.

A direct corollary of this classical result is the following:

Lemma 1. Consider the projective connection (11) on a connected domain D and assume that its Liouville invariant $\lambda$ is nonvanishing on D. Suppose that vector fields $X$ and $Y$ in $\mathfrak{p}($ (1) $)$ satisfy $[X, Y]=X$ and that $X$ is not identically 0 . Then, at every point of a dense open subset of $D$ the vector fields $X$ and $Y$ are linearly independent (and hence fall into the transitive case).

Proof. As we explained in the previous section, $D$ contains a dense open subset on which the pair $(X, Y)$ falls into either the transitive case or the nontransitive case. We need only show that the invariant $\lambda$ vanishes on any open set on which $(X, Y)$ falls into the nontransitive case.

Thus, assume that we are in the nontransitive case, i.e., that one can choose local coordinates $x$ and $y$ such that $X=e^{y} \frac{\partial}{\partial y}$ and $Y=-\frac{\partial}{\partial y}$. Since $Y$ belongs

\footnotetext{
${ }^{10} \mathrm{In}$ fact, it is the lowest order tensorial invariant.
} 
to $\mathfrak{p}(\mathbb{1})$ ), for every solution $y(x)$ of $(\mathbb{1})$ and for every $t \in \mathbb{R}$, we have that $y(x)+t$ is also a solution. It follows without difficulty that the functions $K^{i}$ depend on $x$ only. Substituting $Z:=X=\left(0, e^{y}\right)$ in the equations (3), we obtain $K^{0}=K^{2}=K^{3}=0$, which, together with $K^{1}=K^{1}(x)$, implies, by the formulae (4), that $L_{1}=L_{2}=0$ and hence $\lambda=0$. The Lemma is proved.

\subsubsection{Superintegrable metrics have $\operatorname{dim}(\mathfrak{p})=3$}

Let $g$ be a (pseudo-)Riemannian metric on a connected surface $M$. The geodesic flow of $g$ is then a well-defined flow on $T M$. A function $h: T M \rightarrow \mathbb{R}$ is an integral of the geodesic flow of $g$ if it is constant on the orbits of the geodesic flow of $g$. The necessary and sufficient condition that $h$ be an integral of the geodesic flow of $g$ is that it satisfy the linear first order PDE

$$
\{h, g\}_{g}=0
$$

where $\{,\}_{g}$ is the Poisson bracket on $T M$ that is transferred from the canonical one on $T^{*} M$ via the bundle isomorphism $b_{g}: T M \rightarrow T^{*} M$.

An integral $h$ of the geodesic flow of $g$ is said to be a quadratic integral if it is a quadratic function on each tangent space $T_{p} M$. For example $g$ itself is a quadratic integral of the geodesic flow 11

For quadratic forms $h: T M \rightarrow \mathbb{R}$, the linear equation $\{h, g\}_{g}=0$ is an overdetermined PDE system of finite type. Hence, the quadratic integrals of the geodesic flow of $g$ always form a finite dimensional vector space $\mathcal{I}(g)$. Koenigs [12] proved that, on a connected surface $M$, the dimension of $\mathcal{I}(g)$ is $1,2,3,4$, or 6 . Moreover, if $\operatorname{dim}(\mathcal{I}(g))=6$, then $(M, g)$ is isometric to a connected domain in a surface of constant curvature. The metrics $g$ of nonconstant curvature such that $\operatorname{dim}(\mathcal{I}(g))=4$ (the next highest value) are sometimes said to be superintegrable (or Darboux-suprintegrable) [10.

Two metrics $g$ and $\bar{g}$ on (the same) $D^{2}$ are said to be projectively equivalent if they have the same geodesics, considered as unparameterized curves. The following result connecting projective equivalence and the space $\mathcal{I}(g)$ is proved in Darboux [6, §608] and is based on Darboux' generalization of the work of Dini (see [6, §601]). For recents proofs, see [19, 20, 21, 28.

Theorem 3. Let $g, \bar{g}$ be metrics on $M^{2}$. Then they are projectively equivalent if and only if the function $I: T M^{2} \rightarrow \mathbb{R}$ defined by

$$
I(\xi):=\bar{g}(\xi, \xi)\left(\frac{\operatorname{det}(g)}{\operatorname{det}(\bar{g})}\right)^{2 / 3}
$$

is an integral of the geodesic flow of $g$.

\footnotetext{
${ }^{11}$ The importance of quadratic integrals other than $g$ itself for studying the geodesic flow was recognized long ago. Indeed, it was Jacobi's realization that the geodesic flow of the ellipsoid admitted such an 'extra' quadratic integral that allowed him to integrate the geodesic flow on the ellipsoid.
} 
Remark 3 . Theorem 3 has the corollary that each metric $\bar{g}$ that is projectively equivalent to $g$ is of the form

$$
\bar{g}=\left(\frac{\operatorname{det}(g)}{\operatorname{det}(h)}\right)^{2} h
$$

where $h \in \mathcal{I}(g)$ satisfies the condition $\operatorname{det}(h) / \operatorname{det}(g) \neq 0$.

Another direct corollary of Theorem 3 (see, for example, 26, 28], or Section 2.8 for explanations) is the following result due to Knebelman.

Corollary 1 ([11]). Let two metrics $g$ and $\bar{g}$ on $M^{2}$ be projectively equivalent. Suppose $K=K^{i}$ is a Killing vector field for $g$. Then,

$$
\bar{K}=\left(\frac{\operatorname{det}(\bar{g})}{\operatorname{det}(g)}\right)^{1 / 3} \bar{g}^{-1} g(K):=\left(\frac{\operatorname{det}(\bar{g})}{\operatorname{det}(g)}\right)^{1 / 3} \bar{g}^{\alpha j} g_{\alpha i} K^{i}
$$

is a Killing vector field for $\bar{g}$.

Remark 4 . The mapping $K \mapsto \bar{K}$, though linear, is not always a Lie algebra homomorphism. For example, when $g$ is flat and $\bar{g}$ has nonzero constant curvature, this mapping clearly cannot be a Lie algebra homomorphism.

Koenigs [12] proved that superintegrable metrics always have a Killing vector and classified them [12, Tableau I] 12 Though Koenigs proved this result locally, the global (i.e. on every connected manifold) version of this result follows from his list and from the trivial observation that two Killing vector fields of a metric of nonconstant curvature are proportional.

The existence of a Killing vector field, combined with Corollary 1 and with Theorem 3 gives

Lemma 2. If $g$ on $M^{2}$ is superintegrable, then $\operatorname{dim}(\mathfrak{p}(g))=3$.

Proof. Corollary 1 combined with Theorem 3 says that if $K=K^{i}$ is a Killing vector field of a metric $g$, then for every quadratic integral $F=f_{i j} \xi^{i} \xi^{j}$ (where $\left.f_{i j}=f_{j i}\right)$, the vector field

$$
Z_{F}=\frac{\operatorname{det}(f)}{\operatorname{det}(g)} f^{-1} g(K):=\frac{\operatorname{det}(f)}{\operatorname{det}(g)} f^{i \alpha} g_{\alpha j} K^{j}
$$

is a projective vector field.

Note that, because, on 2-by-2 matrices, the operation $f \mapsto \operatorname{det}(f) f^{-1}$ is linear in $f$, the mapping $F \mapsto Z_{F}$ is a linear map from $\mathcal{I}(g)$ to $\mathfrak{p}(g)$. Its kernel is at most one-dimensional. Indeed, consider $H(\xi)=h_{i j} \xi^{i} \xi^{j}$ with $h_{i j}=h_{j i}$. Since two symmetric 2-by-2 matrices with the same one-dimensional kernel are proportional, the equality $Z_{F}=Z_{H}=0$ implies $F=\lambda H$, or $\lambda F=H$, where

\footnotetext{
${ }^{12}$ One must bear in mind, when consulting [12, particularly the Tables, that Koenigs worked over the complex domain, so that he did not distinguish between the Riemannian and pseudoRiemannian cases. It requires a little work (and careful reading of his notation) to separate out the possible normal forms of superintegrable Riemannian metrics.
} 
$\lambda: M^{2} \rightarrow \mathbb{R}$. If $F, H \in \mathcal{I}(g)$, the function $\lambda$ must also be an integral implying it is constant. Thus, the kernel of the mapping $F \mapsto Z_{F}$ is at most onedimensional. Since $\operatorname{dim}(\mathcal{I}(g))=4$ and the kernel of the linear map $F \mapsto Z_{F}$ (from $\mathcal{I}(g)$ to $\mathfrak{p}(g))$ is at most one-dimensional, $\operatorname{dim}(\mathfrak{p}(g)) \geq 3$. Since as we recalled in Section 2.2.2 metrics of nonconstant curvature have $\operatorname{dim}(\mathfrak{p}) \leq 3$, we obtain $\operatorname{dim}(\mathfrak{p}(g))=3$. Lemma 2 is proved.

Remark 5. For use in Section 2.8 let us note that the kernel of the mapping $F \mapsto Z_{F}$ from the proof of Lemma 2 is the linear hull of the function $F_{K}(\xi):=$ $\left(g_{i j} K^{i} \xi^{j}\right)^{2} \in \mathcal{I}(g)$, which can be checked by direct calculations.

Corollary 2. If the space of metrics having a given projective connection is more than 3-dimensional, then each metric $g$ from this space has $\operatorname{dim}(\mathfrak{p}(g))>2$.

Proof. Because of Theorem 3 and Remark 3, superintegrable metrics are those admitting a 4-parameter family of projectively equivalent metrics. By Lemma2, they have $\operatorname{dim}(\mathfrak{p})>2$. Corollary 2 is proved.

Corollary 2 is actually what we will need from this section. Let us note that the converse statement is also true.

Corollary 3. A metric $g$ of nonconstant curvature on connected $M^{2}$ such that $\operatorname{dim}(\mathfrak{p})=3$ is superintegrable.

We will prove Corollary 3 at the very end of the paper, in Section 2.8 .

\subsection{Projective connections admitting two infinitesimal symmetries}

Lemma 3. Let the projective connection (11) admit two infinitesimal symmetries that are linearly independent at the point $p$. Then there exists a coordinate system $x, y$ in a neighborhood of $p$ such that the vector fields $X:=(0,1)$ and $Y:=$ $(1, y)$ belong to $\mathfrak{p}(\mathbf{1})$ ). In such a coordinate system, the projective connection (1D) has the form

$$
y^{\prime \prime}(x)=A e^{x}+B y^{\prime}(x)+C e^{-x}\left(y^{\prime}(x)\right)^{2}+D e^{-2 x}\left(y^{\prime}(x)\right)^{3},
$$

where $A, B, C, D$ are constants. Moreover, such a coordinate system can be chosen so that one of the following conditions holds:

1. $D \neq 0, C=0$,

2. $D=0, C \neq 0, B=0$, or

3. $D=C=B=A=0$.

The vector fields $(0,1)$ and $(1, y)$ are always infinitesimal symmetries of the projective connection (7). Therefore, $\operatorname{dim} \mathfrak{p}((17)) \geq 2$. For certain values of $A$, $B, C$, and $D$, one can have $\operatorname{dim} \mathfrak{p}((7))>2$. The following lemma describes all such values of $A, B, C$, and $D$. 
Lemma 4. The following statements hold

1. The projective connection $y^{\prime \prime}(x)=A e^{x}+B y^{\prime}(x)+D e^{-2 x}\left(y^{\prime}(x)\right)^{3}$ with $D \neq 0$ admits a infinitesimal symmetry that is not a linear combination of the vector fields $(0,1)$ and $(1, y)$ if and only if $A=0$ and $B \in\{1 / 2,2\}$. Moreover, if $A=0$ and $B=2$, then the projective connection is flat. If $A=0$ and $B=1 / 2$, every infinitesimal symmetry is a linear combination of the vector fields $(0,1),(1, y)$, and $\left(y, y^{2} / 2\right)$.

2. For $C \neq 0$ the algebra of infinitesimal symmetries of the projective connection $y^{\prime \prime}(x)=A e^{x}+C e^{-x}\left(y^{\prime}(x)\right)^{2}$ is spanned by the vector fields $(0,1)$ and $(1, y)$.

Proof of Lemmas 3. 4. These lemmas follow from the results of Beltrami, Lie, Cartan, and Tresse that we recalled in Section 2.2 and are not new, see for example [13, 27. We give their proofs to make this article self-contained.

We assume that the projective connection is not flat. By Lemma 1 in a certain coordinate system $u=(1, y)$ and $v=(0,1)$ are projective vector fields of this connection.

It is easy to construct the flows of these vector fields. Indeed, the flow of $v$ is $\Psi_{\tau}(x, y)=(x, y+\tau)$. The flow of $u$ is $\Phi_{\tau}(x, y)=\left(x+\tau, e^{\tau} y\right)$. Since the flow of the vector field $u=(0,1)$ preserves the geodesics, for every solution $y(x)$ and for every $\tau$ the function $y(x)+\tau$ is also a solution of the equation (1). Thus, the coefficients $K^{0}, K^{1}, K^{2}, K^{3}$ are independent of $y$.

Similarly, since the flow of the vector field $u=(1, y)$ preserves the geodesics, for every solution $y(x)$ and for every $\tau$ the function $e^{\tau} y(x+\tau)$ is a solution of the equation (11). Hence,

$$
e^{\tau} y^{\prime \prime}(x+\tau)=K^{0}+e^{\tau} K^{1} y^{\prime}(x+\tau)+e^{2 \tau} K^{2}\left(y^{\prime}(x+\tau)\right)^{2}+e^{3 \tau} K^{3}\left(y^{\prime}(x+\tau)\right)^{3} .
$$

Thus, the projective connection has the form (77).

Now let us show that we can change coordinates so that the constants $A, B, C, D$ will satisfy conditions 1,2 , or 3 . Consider the following new coordinate system: $x_{\text {new }}:=x_{\text {old }}, y_{\text {new }}:=y_{\text {old }}+\alpha e^{x_{\text {old }}}$. In these new coordinates, (7) becomes

$$
\begin{aligned}
y^{\prime \prime}(x)= & A_{\text {new }} e^{x}+B_{\text {new }} y^{\prime}(x)+C_{\text {new }} e^{-x}\left(y^{\prime}(x)\right)^{2}+D_{\text {new }} e^{-2 x}\left(y^{\prime}(x)\right)^{3} \\
= & \left(A-\alpha+B \alpha+C \alpha^{2}+D \alpha^{3}\right) e^{x}+\left(3 D \alpha^{2}+2 C \alpha+B\right) y^{\prime}(x) \\
& +(3 D \alpha+C) e^{-x}\left(y^{\prime}(x)\right)^{2}+D e^{-2 x}\left(y^{\prime}(x)\right)^{3} .
\end{aligned}
$$

This new equation has the same form as (7) and therefore the vector fields $(0,1),(1, y)$ are infinitesimal symmetries of this equation (which is not surprising because the coordinate change preserves the vector field $(0,1)$ and sends the vector field $(1, y)$ to a linear combination of $(1, y)$ and $(0,1))$.

We see that if $D \neq 0$, then by the appropriate choice of $\alpha$ we can make $C_{\text {new }}=0$ so that the constants $A_{\text {new }}, B_{\text {new }}, C_{\text {new }}, D_{\text {new }}$ will satisfy the first case from Lemma 3. Similarly, if $D=0$ and $C \neq 0$, then by the appropriate 
choice of $\alpha$ we can make $B_{\text {new }}=0$ so that the constants $A_{\text {new }}, B_{\text {new }}, C_{\text {new }}, D_{\text {new }}$ will satisfy the second case of Lemma 3 . If $D=C=0$, then both Cartan invariants (recalled in Section 2.2.3) vanish and therefore, in some coordinate system, $A=B=C=D=0$. Lemma 3 is proved.

Let us now prove Lemma 4 Suppose first that the algebra $\mathfrak{p}(\sqrt{77})$ is 8dimensional. Then, by the result of Lie, Liouville, Tresse and Cartan recalled in Section 2.2.3, we have $L_{1}=L_{2}=0$. Substituting the coefficients of the connection in the formulae for $L_{2}$ and $L_{1}$, we obtain

$$
\begin{aligned}
6 D(B-2)-2 C^{2} & =0 \\
C+9 A D-B C & =0 .
\end{aligned}
$$

Finally, if $B=D=0$ then the second equation implies $C=0$. If $D \neq 0$ and $C=0$ then the equations imply $A=0, B=2$. Lemma 4 is proved under the assumption that the algebra of infinitesimal symmetries is 8-dimensional.

Now suppose the projective connection (7) has a three-dimensional algebra of infinitesimal symmetries. Then, it is isomorphic to $\mathfrak{s l}(2, \mathbb{R})$, see Section 2.2.2. and therefore is generated by three vector fields $X, Y, Z$ satisfying

$$
[X, Y]=X, \quad[X, Z]=Y, \quad[Y, Z]=Z .
$$

Without loss of generality, in view of Lemma 1, we can assume that $X=(0,1)$ and $Y=(1, y)$. Indeed, the vector fields $(0,1)$ and $(1, y)$ satisfy $[X, Y]=X$ and therefore form a Borel subalgebra in the algebra $\mathfrak{s l}(2, \mathbb{R})$ of infinitesimal symmetries, and all Borel subalgebras of $\mathfrak{s l}(2, \mathbb{R})$ are isomorphic. Then, the vector field $Z$ must satisfy the conditions

$$
[X, Z]=Y, \quad[Y, Z]=Z .
$$

These conditions are equivalent to the following system of partial differential equations on the components $Z^{1}, Z^{2}$ of the vector field $Z$.

$$
\left.\begin{array}{rl}
\frac{\partial Z^{1}}{\partial y}-1 & =0 \\
\frac{\partial Z^{2}}{\partial y}-y & =0 \\
\frac{\partial Z^{1}}{\partial x}+y \frac{\partial Z^{1}}{\partial y}-Z^{1} & =0 \\
\frac{\partial Z^{2}}{\partial x}+y \frac{\partial Z^{2}}{\partial y}-2 Z^{2} & =0
\end{array}\right\} .
$$

Solving the system we obtain $Z=\left(Z^{1}, Z^{2}\right)=\left(y+C_{1} e^{x}, \frac{y^{2}}{2}+C_{2} e^{2 x}\right)$, where $C_{1}, C_{2}$ are constants. But if such a vector $Z$ is an infinitesimal symmetry, then the equations (3) corresponding to the connection from the second statement of Lemma 4 imply the equality

$$
-C e^{-x}=C_{1} C+1=\left(3 A+4 C C_{2}+C_{1}\right) e^{x}=\left(4 C_{2}-3 C_{1} A\right) e^{2 x}=0,
$$

which is incompatible. Thus, the second statement of Lemma 4 is proved. Similarly, the equations (3) corresponding to the connections from the second 
statement of Lemma 4 imply the equality

$3 C_{1} D e^{-x}=\left(1-2 B-6 D C_{2}\right)=-\left(C_{1} B+C_{1}+3 A\right) e^{x}=\left(4 C_{2}-3 C_{1} A-2 B C_{2}\right) e^{2 x}=0$.

Thus, $\left\{C_{1}=0, C_{2}=-\frac{1}{2 D}, A=0, B=2\right\}$, or $\left\{C_{1}=0, C_{2}=0, A=0, B=\frac{1}{2}\right\}$.

As we explained above, the first case corresponds to a connection with 8-

dimensional space of infinitesimal symmetries. Lemma 4 is proved.

\subsection{When does a given projective connection come from a metric?}

Consider a projective connection

$$
y^{\prime \prime}(x)=K^{0}(x, y)+K^{1}(x, y) y^{\prime}(x)+K^{2}(x, y)\left(y^{\prime}(x)\right)^{2}+K^{3}(x, y)\left(y^{\prime}(x)\right)^{3} .
$$

We ask when there exists a metric with this projective connection and, if any exist, how to find them. Lemma 5 below, due to R. Liouville [18, Chapter III, $\S \mathrm{XI}]$, gives a useful tool to answer this question.

Fix a coordinate system $x, y$ (which, for notational ease, we shall sometimes refer to as $\left.\left(x^{1}, x^{2}\right)\right)$. Given a metric $g=E d x^{2}+2 F d x d y+G d y^{2}$, construct the symmetric nondegenerate matrix

$$
a=\left(\begin{array}{ll}
a_{11} & a_{12} \\
a_{12} & a_{22}
\end{array}\right):=\operatorname{det}(g)^{-2 / 3} g=\frac{1}{\left(E G-F^{2}\right)^{2 / 3}}\left(\begin{array}{cc}
E & F \\
F & G
\end{array}\right) .
$$

Lemma 5 ([18]). The projective connection of the metric $g$ is (8) if and only if the entries of the matrix a satisfy the linear PDE system

$$
\left.\begin{array}{rl}
a_{11 x}-\frac{2}{3} K^{1} a_{11}+2 K^{0} a_{12} & =0 \\
a_{11 y}+2 a_{12 x}-\frac{4}{3} K^{2} a_{11}+\frac{2}{3} K^{1} a_{12}+2 K^{0} a_{22} & =0 \\
2 a_{12 y}+a_{22 x}-2 K^{3} a_{11}-\frac{2}{3} K^{2} a_{12}+\frac{4}{3} K^{1} a_{22} & =0 \\
a_{22 y}-2 K^{3} a_{12}+\frac{2}{3} K^{2} a_{22} & =0
\end{array}\right\}
$$

Remark 6. Before proving the lemma, let us explain how to define the system (10) more conceptually. Consider the jet space $J_{1}^{1}$ (since we are working locally we can think of $J_{1}^{1}$ as $\mathbb{R}^{3}$ with coordinates $\left.\left(x, y, y_{x}\right)\right)$. Consider the function

$$
F: J^{1} \rightarrow \mathbb{R}, \quad F\left(x, y, y_{x}\right):=a_{11}+2 a_{12} y_{x}+a_{22} y_{x}^{2},
$$

(i.e., the function $F$ is the square of the length of the vector $\left(1, y_{x}\right)$ in the quadratic form corresponding to the matrix $a$ ), the function

$$
\alpha\left(x, y, y_{x}\right):=\frac{\partial y_{x x}}{\partial y_{x}}:=K^{1}+2 K^{2}\left(y_{x}\right)+3 K^{3}\left(y_{x}\right)^{2},
$$


and the 'total derivative' $D_{x}$ restricted to (1)

$$
D_{x}:=\frac{\partial}{\partial x}+y_{x} \frac{\partial}{\partial y}+\left(K^{0}+K^{1} y_{x}+K^{2}\left(y_{x}\right)^{2}+K^{3}\left(y_{x}\right)^{3}\right) \frac{\partial}{\partial y_{x}}
$$

Then the system (10) is equivalent to the equation $D_{x}(F)=\frac{2}{3} F \alpha$. More precisely, the equation $D_{x}(F)-\frac{2}{3} F \alpha=0$ is polynomial of third degree in $y_{x}$ and the equations from the system (10) are coefficients of this polynomial.

Remark 7. The proof that we will give below is essentially Liouville's proof, just in different notation.

At first glance, the change of variables (9) that renders the obvious equations on $E, F$, and $G$ linear in the unknowns $a_{i j}$ seems miraculous 13 However, as Liouville himself noted, the occurrence of the matrix $a$ via (9) in Lemma 5] can be motivated by considering its link with the linear equation for quadratic first integrals, as we will now explain.

If the projective connection (1) were known to be that of a metric associated to a quadratic function $h: T M \rightarrow \mathbb{R}$, then, by Theorem 3 , a metric $g$ would have the same geodesics as $h$ (and hence have (1) as its projective connection) if and only if it were to satisfy

$$
\left\{\left(\frac{\operatorname{det}(h)}{\operatorname{det}(g)}\right)^{\frac{2}{3}} g, h\right\}_{h}=0
$$

where $\{,\}_{h}$ is the Poisson bracket transplanted from $T^{*} M$ to $T M$ by the bundle isomorphism $b_{h}: T M \rightarrow T^{*} M$. (Note that, although $\operatorname{det}(g) \operatorname{depends~on~a~}$ choice of coordinates, the ratio $\frac{\operatorname{det}(h)}{\operatorname{det}(g)}$ does not, so (11) is actually a coordinateindependent equation.)

The equation (11) is visibly linear in $a=g / \operatorname{det}(g)^{2 / 3}$, which motivates expressing the projective geodesic condition for $g$ in terms of $a$.

Proof. We will first derive a system of PDE on the components of the metric $g$ whose solvability corresponds to the existence of metric with the projective connection (8) and then show that this system is equivalent to the system (10).

First of all, it is easy to obtain all symmetric affine connections whose projective connection is (8). Indeed, by the definition of the projective connection corresponding to an affine connection (see Section 2.2.1), the components of the symmetric affine connection should satisfy the system of four linear equations

$$
K^{0}=-\Gamma_{11}^{2}, \quad K^{1}=\Gamma_{11}^{1}-2 \Gamma_{12}^{2}, \quad K^{2}=-\Gamma_{22}^{2}+2 \Gamma_{12}^{1}, \quad K^{3}=\Gamma_{22}^{1} .
$$

Solving this system for the $\Gamma_{j k}^{i}$ gives

$$
\begin{array}{lll}
\Gamma_{11}^{2}=-K^{0}, & \Gamma_{11}^{1}=K^{1}+2 p_{1}, & \Gamma_{21}^{1}=\Gamma_{12}^{1}=p_{2} \\
\Gamma_{22}^{1}=K^{3}, & \Gamma_{22}^{2}=-K^{2}+2 p_{2}, & \Gamma_{21}^{2}=\Gamma_{12}^{2}=p_{1}
\end{array}
$$

\footnotetext{
${ }^{13}$ Indeed, Liouville himself seems to have regarded this linearity as a remarkably lucky circumstance.
} 
where $p_{1}$ and $p_{2}$ are functions on $D^{2}$.

By definition, $\Gamma_{j k}^{i}$ is the Levi-Civita connection of the unknown metric $g=$ $g_{i j}$, if and only if the covariant derivative $g_{i j, k}$ in this connection vanishes. The standard formula

$$
g_{i j, k}=\frac{\partial g_{i j}}{\partial x_{k}}-\sum_{\alpha=1}^{2}\left(\Gamma_{j k}^{\alpha} g_{i \alpha}+\Gamma_{i k}^{\alpha} g_{\alpha j}\right)
$$

then gives us the following six first order PDE on five unknown functions, which are the components $E, F$, and $G$ of the metric and the functions $p_{1}, p_{2}$.

$$
\left.\begin{array}{rl}
\frac{\partial E}{\partial x}-2 K^{1} E+2 K^{0} F-4 E p_{1} & =0 \\
\frac{\partial E}{\partial y}-2 p_{2} E-2 p_{1} F & =0 \\
\frac{\partial F}{\partial y}-K^{3} E+K^{2} F-3 p_{2} F-p_{1} G & =0 \\
\frac{\partial F}{\partial x}-K^{1} F+K^{0} G-3 p_{1} F-p_{2} E & =0 \\
\frac{\partial G}{\partial x}-2 p_{2} F-2 p_{1} G & =0 \\
\frac{\partial G}{\partial y}-2 K^{3} F+2 K^{2} G-4 G p_{2} & =0
\end{array}\right\}
$$

Thus, the metric $g=\left(\begin{array}{cc}E & F \\ F & G\end{array}\right)$ has projective connection (8) if and only if there exist functions $p_{1} p_{2}$ such that $E, F, G, p_{1}$, and $p_{2}$ satisfy the system (12).

Assuming that $E G-F^{2} \neq 0$, solving the second and fifth equation of (12) for $p_{1}$ and $p_{2}$, and then substituting the result into the remaining equations, we obtain a system of four first order PDE for the three functions $E, F$, and $G$, whose solvability is equivalent to the existence of a metric with the projective connection (8).

Now making the substitution

$$
\left(\begin{array}{cc}
E & F \\
F & G
\end{array}\right)=\frac{a}{\operatorname{det}(a)^{2}}=\left(\begin{array}{ll}
\frac{a_{11}}{\left(a_{11} a_{22}-a_{12}^{2}\right)^{2}} & \frac{a_{12}}{\left(a_{11} a_{22}-a_{12}^{2}\right)^{2}} \\
\frac{a_{12}}{\left(a_{11} a_{22}-a_{12}^{2}\right)^{2}} & \frac{a_{22}}{\left(a_{11} a_{22}-a_{12}^{2}\right)^{2}}
\end{array}\right),
$$

(which is equivalent to (9)) into these equations yields, after some computation, the system (10). Lemma 5 is proved.

\subsection{Reducing the system (10) to a system of ODE and solving it}

In this section we assume that our metric $g$ admits precisely two linearly independent projective vector fields. Then, by Lemma 3 in some coordinate system near this point the projective connection of this metric is given as

$$
y^{\prime \prime}(x)=A e^{x}+B y^{\prime}(x)+C e^{-x}\left(y^{\prime}(x)\right)^{2}+D e^{-2 x}\left(y^{\prime}(x)\right)^{3} .
$$


By Lemma 5 the components $a_{11}, a_{12}, a_{22}$ of the matrix $a$ constructed for the metric $g$ by the formula (9) satisfy the system (10). Meanwhile, the coefficients of (10), considered as a linear first order system for $a$, do not depend on $y$ because they are either constants or constant multiples of the $K^{i}$, which are independent of $y$ for the projective connection (13).

Thus, if $a=\left(a_{i j}\right)$ is a solution of (10), then $\frac{\partial a}{\partial y}, \frac{\partial^{2} a}{\partial y^{2}}$, and $\frac{\partial^{3} a}{\partial y^{3}}$ are also solutions. However, by Corollary 2, our assumption that the space of projective vector fields is precisely two-dimensional implies that the space of metrics having (13) as projective connection has dimension at most three. Thus, there exist constants $\lambda_{0}, \lambda_{1}, \lambda_{2}, \lambda_{3}$ (with at least one of $\lambda_{1}, \lambda_{2}, \lambda_{3}$ being nonzero) such that

$$
\lambda_{0} a+\lambda_{1} \frac{\partial a}{\partial y}+\lambda_{2} \frac{\partial^{2} a}{\partial y^{2}}+\lambda_{3} \frac{\partial^{3} a}{\partial y^{3}}=0 .
$$

Hence, the functions $a_{11}, a_{12}$, and $a_{22}$ all satisfy some linear ordinary differential equation of at most third order with constant coefficients.

Depending on the multiplicity of the roots of the characteristic polynomial of this equation, the functions $a_{11}, a_{12}$, and $a_{22}$ are given by one of the following formulae.

Case 1: $a_{11}=c_{01} e^{\alpha_{1} y}+c_{02} e^{\alpha_{2} y}+c_{03} e^{\alpha_{3} y}, \quad a_{12}=2 c_{11} e^{\alpha_{1} y}+2 c_{12} e^{\alpha_{2} y}+$ $2 c_{13} e^{\alpha_{3} y}, \quad a_{22}=c_{21} e^{\alpha_{1} y}+c_{22} e^{\alpha_{2} y}+c_{23} e^{\alpha_{3} y}$, where $\alpha_{1} \in \mathbb{R}, \alpha_{2}, \alpha_{3} \in \mathbb{C}$ are mutually different constants and $c_{i j}$ are functions of $x$.

Case 2: $\quad a_{11}=c_{01} e^{\alpha_{1} y}+c_{02} e^{\alpha_{2} y}+c_{03} y e^{\alpha_{2} y}, \quad a_{12}=2 c_{11} e^{\alpha_{1} y}+$ $2 c_{12} e^{\alpha_{2} y}+2 c_{13} y e^{\alpha_{2} y}, \quad a_{22}=c_{21} e^{\alpha_{1} y}+c_{22} e^{\alpha_{2} y}+c_{23} y e^{\alpha_{2} y}$, where $\alpha_{1} \in$ $\mathbb{R}, \alpha_{2} \in \mathbb{C}$ are mutually different constants and $c_{i j}$ are functions of $x$.

Case 3: $a_{11}=c_{01} e^{\alpha y}+c_{02} y e^{\alpha y}+c_{03} y^{2} e^{\alpha y}, \quad a_{12}=2 c_{11} e^{\alpha y}+2 c_{12} y e^{\alpha y}+$ $2 c_{13} y^{2} e^{\alpha y}, \quad a_{22}=c_{21} e^{\alpha y}+c_{22} y e^{\alpha y}+c_{23} y e^{\alpha y}$, where $\alpha \in \mathbb{R}$ is a constant and $c_{i j}$ are functions of $x$.

In Cases 1 and 2, we allow also complex-conjugated $\alpha_{i}$.

Substituting the above ansatz for $a_{i j}$ in the equations (10) and using that the functions $y^{j} e^{\alpha_{i} y}$ are linearly independent for different $j$ and $\alpha_{i}$, we see that the system (10) is equivalent to the following system of ODE on the functions $c_{i j}$. The system contains 9 ordinary differential equations of the form

$$
\frac{d}{d x} c=M c,
$$

where $c$ is the column with entries $\left(c_{01}, c_{02}, c_{03}, c_{11}, c_{12}, c_{13}, c_{21}, c_{22}, c_{23}\right)$ and $M$

\begin{tabular}{|c|c|c|c|c|c|c|c|c|}
\hline$\frac{2}{3} B$ & 0 & 0 & $-A e^{x}$ & 0 & 0 & 0 & 0 & 0 \\
\hline 0 & $\frac{2}{3} B$ & 0 & 0 & $-A e^{x}$ & 0 & 0 & 0 & 0 \\
\hline 0 & 0 & $\frac{2}{3} B$ & 0 & 0 & $-A e^{x}$ & 0 & 0 & 0 \\
\hline$\frac{4}{3} C e^{-x}-\alpha_{1}$ & $k_{1}$ & 0 & $-\frac{1}{3} B$ & 0 & 0 & $-2 A e^{x}$ & 0 & 0 \\
\hline 0 & $\frac{4}{3} C e^{-x}-\alpha_{2}$ & $k_{2}$ & 0 & $-\frac{1}{3} B$ & 0 & 0 & $-2 A e^{x}$ & 0 \\
\hline 0 & 0 & $\frac{4}{3} C e^{-x}-\alpha_{3}$ & 0 & 0 & $-\frac{1}{3} B$ & 0 & 0 & $-2 A e^{x}$ \\
\hline $2 D e^{-2 x}$ & 0 & 0 & $\frac{1}{3} C e^{-x}-\alpha_{1}$ & $k_{1}$ & 0 & $-\frac{4}{3} B$ & 0 & 0 \\
\hline 0 & $2 D e^{-2 x}$ & 0 & 0 & $\frac{1}{3} C e^{-x}-\alpha_{2}$ & $k_{2}$ & $\begin{array}{l}3 \\
0\end{array}$ & $-\frac{4}{3} B$ & 0 \\
\hline 0 & 0 & $2 D e^{-2 x}$ & 0 & 0 & $\frac{1}{3} C e^{-x}-\alpha_{3}$ & 0 & 0 & $-\frac{4}{3} B$ \\
\hline
\end{tabular}
is the following 9-by-9 matrix, 
and three linear equations on $c_{i j}$, which can be written as

$$
\left(\begin{array}{ccc}
\alpha_{1}+\frac{2}{3} C e^{-x} & k_{1} & 0 \\
0 & \alpha_{2}+\frac{2}{3} C e^{-x} & k_{2} \\
0 & 0 & \alpha_{3}+\frac{2}{3} C e^{-x}
\end{array}\right)\left(\begin{array}{l}
c_{21} \\
c_{22} \\
c_{23}
\end{array}\right)=D e^{-2 x}\left(\begin{array}{l}
c_{11} \\
c_{12} \\
c_{13}
\end{array}\right)
$$

where, the parameters $\alpha_{i} \in \mathbb{C}, k_{i} \in\{0,-1,-2\}$ satisfy

1. $\alpha_{1} \neq \alpha_{2} \neq \alpha_{3} \neq \alpha_{1}, k_{1}=k_{2}=0$ for the case 1 ,

2. $\alpha_{1} \neq \alpha_{2}=\alpha_{3}, k_{1}=0, k_{2}=-1$ for the case 2 ,

3. $\alpha_{1}=\alpha_{2}=\alpha_{3}, k_{1}=-1 k_{2}=-2$ for the case 3 .

Thus, every metric admitting precisely two projective vector fields comes from the solution of the system above. Note that in view of Lemmas 3 and 4 we can assume

(a) $C \neq 0$ and $D=B=0$,

(b) or $D \neq 0, C=0$, and if $A=0$, then $B \neq 2$ and $B \neq 1 / 2$.

Lemma 6. Consider the above system of ODE corresponding to one of the cases 1,2,3. Then, the following holds.

1. If the condition (a) holds, the system admits the trivial solution $c_{i j} \equiv 0$ only.

2. If the condition (a) holds and $A \neq 0$, then the system admits the trivial solution $c_{i j} \equiv 0$ only.

3. If the condition (b) holds and $A=0$, then

(*) for $B \neq 1$ the general solution of the system corresponds to

$$
a=\lambda\left(\begin{array}{cc}
e^{\frac{2}{3} B x} & 0 \\
0 & \left(D \frac{e^{2(B-1) x}}{B-1}+H\right) e^{-\frac{4}{3} B x}
\end{array}\right)
$$

where $H \in \mathbb{R}$, or

(**) for $B=1$ the general solution of the system corresponds to

$$
a=\lambda\left(\begin{array}{cc}
e^{\frac{2}{3} x} & 0 \\
0 & (2 D x+H) e^{-\frac{4}{3} x}
\end{array}\right)
$$

where $H \in \mathbb{R}$.

Remark 8. By (9) we have $g=a /(\operatorname{det}(a))^{2}$. Then, the solution (16) for $H=$ $0(H \neq 0$, respectively) corresponds to the metric (1a) (to the metric (1b), respectively) from Theorem 11, after the coordinate change $\left(x_{\text {new }}, y_{\text {new }}\right)=(x+$ $\left.c_{1}, c_{2} y\right)$ for the appropriate $c_{1}, c_{2} \in \mathbb{R}$ and by setting $b:=2(1-B)$. Recall that $B \neq \frac{1}{2}, 1,2$ by assumptions, which implies $b \neq 1,0,-2$. Similarly, the solution (17) corresponds to the metric (1c) from Theorem 1 
Sketch of the proof of Lemma [6. Before giving detailed calculations (below), let us explain the ideas staying behind, and one more proof which is actually simpler than one staying below, if we allow calculations done with the help of modern computer algebra programs.

The 'computer algebra' proof is very straightforward: the system (14) can be explicitly solved (Maple does it), the general solution depends on 9 constants, say $C_{1}, \ldots, C_{9}$. Substituting the solution in the equations (15), we obtain algebraic relations on the constants $C_{1}, \ldots C_{9}$ and $A, B, C, D$. Analyzing these relations, one obtains the Lemma.

Now let us explain a trick which allowed us to give a hand-written ( = without the help of computer algebra programs) proof. Differentiate the equations (15) by $x$ and substitute the values of $\frac{\mathrm{d}}{\mathrm{d} x} c_{i j}$ given by (14) inside. We obtain three new linear equations on $c_{i j}$. Repeat the procedure: Differentiate these new equations by $x$ and substitute the values of $\frac{\mathrm{d}}{\mathrm{d} x} c_{i j}$ given by (14) inside. We obtain another triple of linear equations on $c_{i j}$. These two triples of equations together with (15) gives us 9 linear equations on 9 functions $c_{i j}$. The corresponding 9-by-9 matrix can be explicitly constructed. One immediately sees that under assumptions of the first and the second statements of the Lemma this matrix is nondegenerate almost everywhere implying $c_{i j} \equiv 0$. Under assumptions of the third statement of the Lemma, the matrix has rank 7 implying that the system (14) can be reduced to two ODE, which can be explicitly solved. Their solution gives us the third statement of the Lemma.

Proof of Lemma 6. Assume $B=D=0, C \neq 0$. Then, the equations (15) imply $c_{21} \equiv c_{22} \equiv c_{23} \equiv 0$. Substituting this in the last three equations of (14), we obtain the system

$$
\left(\begin{array}{ccc}
\frac{1}{3} C e^{-x}-\alpha_{1} & k_{1} & 0 \\
0 & \frac{1}{3} C e^{-x}-\alpha_{2} & k_{2} \\
0 & 0 & \frac{1}{3} C e^{-x}-\alpha_{3}
\end{array}\right)\left(\begin{array}{l}
c_{11} \\
c_{12} \\
c_{13}
\end{array}\right)=\left(\begin{array}{l}
0 \\
0 \\
0
\end{array}\right),
$$

which evidently implies $c_{11} \equiv c_{12} \equiv c_{13} \equiv 0$. Substituting $c_{11} \equiv c_{12} \equiv c_{13} \equiv$ $c_{21} \equiv c_{22} \equiv c_{23} \equiv 0$ in the fourth, fifth and sixth equations of (14), we obtain the system

$$
\left(\begin{array}{ccc}
\frac{4}{3} C e^{-x}-\alpha_{1} & k_{1} & 0 \\
0 & \frac{4}{3} C e^{-x}-\alpha_{2} & k_{2} \\
0 & 0 & \frac{4}{3} C e^{-x}-\alpha_{3}
\end{array}\right)\left(\begin{array}{l}
c_{01} \\
c_{02} \\
c_{03}
\end{array}\right)=\left(\begin{array}{l}
0 \\
0 \\
0
\end{array}\right),
$$

implying $c_{01} \equiv c_{02} \equiv c_{03} \equiv 0$. Thus, the system admits only trivial solutions. The first statement of the lemma is proved.

Now let us prove the second statement of the lemma. We assume $D \neq 0$, $C=0, A \neq 0$ and show that the system admits only trivial solutions.

Let us take the thrid, the sixth and the ninth equations of (14) and the last equation of (15). We see that these equations contain only $c_{03}, c_{13}, c_{23}$ and can be written as follows. 


$$
\begin{gathered}
\frac{\mathrm{d}}{\mathrm{dx}}\left(\begin{array}{c}
c_{03} \\
c_{13} \\
c_{23}
\end{array}\right)=\left(\begin{array}{ccc}
\frac{2}{3} B & -A e^{x} & 0 \\
-\alpha_{3} & -\frac{1}{3} B & -2 A e^{x} \\
2 D e^{-2 x} & -\alpha_{3} & -\frac{4}{3} B
\end{array}\right)\left(\begin{array}{c}
c_{03} \\
c_{13} \\
c_{23}
\end{array}\right) \\
\alpha_{3} c_{23}=D e^{-2 x} c_{13}
\end{gathered}
$$

Take the equation (19), differentiate it by $x$ (we obtain a linear equation in $c_{i 3}$ and $\frac{d}{d x} c_{i 3}$ ), and substitute the values of $\frac{d}{d x} c_{i 3}$ given by (18) inside. We obtain new linear equations in $c_{i 3}$. Let us play the same game with this new equation, i.e., differentiate by $x$ and substitute the values of $\frac{d}{d x} c_{i 3}$ inside. We obtain one more linear equation in $c_{i 3}$. The equation (19) together with two new obtained equations can be written as $M_{1} c_{1}=0$, where $c_{1}$ is the column with components $c_{03}, c_{13}, c_{23}$ and $M_{1}$ is the following 3-by-3 matrix

\begin{tabular}{|c|c|c|}
\hline 0 & $-e^{-2 x} D$ & $\alpha_{3}$ \\
\hline $3 \alpha_{3} e^{-2 x} D$ & $-\alpha_{3}{ }^{2}+2 e^{-2 x} D+1 / 3 e^{-2 x} D B$ & $-4 / 3 \alpha_{3} B+2 e^{-x} D A$ \\
\hline$\alpha_{3}{ }^{3}-\alpha_{3} B e^{-2 x} D-$ & $5 / 3 \alpha_{3}{ }^{2} B-5 e^{-x} D A \alpha_{3}-4 e^{-2 x} D-$ & $2 \alpha_{3}{ }^{2} A e^{x}+\frac{16}{9} \alpha_{3} B^{2}$ \\
$8 \alpha_{3} e^{-2 x} D+4 e^{-3 x} D^{2} A$ & $4 / 3 e^{-2 x} D B-1 / 9 e^{-2 x} D B^{2}$ & $-6 e^{-x} D A-10 / 3 e^{-x} D A B$ \\
\hline
\end{tabular}

We see that if $A \neq 0$, then the determinant of the matrix is nonzero at almost every point (because the term at $e^{-6 x}$ is equal to $-8 D^{4} A^{2}$ ). Then, $c_{03} \equiv c_{13} \equiv c_{23} \equiv 0$.

Let us substitute $c_{03} \equiv c_{13} \equiv c_{23} \equiv 0$ in the second, fifth and eighth equations of (14) and in the second equation of (15). We obtain the equation of the form

$$
\begin{gathered}
\frac{\mathrm{d}}{\mathrm{dx}}\left(\begin{array}{c}
c_{02} \\
c_{12} \\
c_{22}
\end{array}\right)=\left(\begin{array}{ccc}
\frac{2}{3} B & -A e^{x} & 0 \\
-\alpha_{2} & -\frac{1}{3} B & -2 A e^{x} \\
2 D e^{-2 x} & -\alpha_{2} & -\frac{4}{3} B
\end{array}\right)\left(\begin{array}{c}
c_{02} \\
c_{12} \\
c_{22}
\end{array}\right), \\
\alpha_{2} c_{22}=D e^{-2 x} c_{12} .
\end{gathered}
$$

We see that these equations are very similar to (18) and (19) (the only difference is $\alpha_{2}$ in the place of $\alpha_{3}$ ). Arguing as above, we obtain $c_{02} \equiv c_{12} \equiv c_{22} \equiv$ 0 . Substituting $c_{03} \equiv c_{13} \equiv c_{23} \equiv c_{02} \equiv c_{12} \equiv c_{22} \equiv 0$ in the first, fourth and seventh equations of (14) and in the first equation of (15), we obtain the equations in the form

$$
\begin{gathered}
\frac{\mathrm{d}}{\mathrm{dx}}\left(\begin{array}{c}
c_{01} \\
c_{11} \\
c_{21}
\end{array}\right)=\left(\begin{array}{ccc}
\frac{2}{3} B & -A e^{x} & 0 \\
-\alpha_{1} & -\frac{1}{3} B & -2 A e^{x} \\
2 D e^{-2 x} & -\alpha_{1} & -\frac{4}{3} B
\end{array}\right)\left(\begin{array}{c}
c_{01} \\
c_{11} \\
c_{21}
\end{array}\right), \\
\alpha_{1} c_{21}=D e^{-2 x} c_{11} .
\end{gathered}
$$

We see that these equations are again very similar to (18) and (19) (the only difference is $\alpha_{1}$ in the place of $\alpha_{3}$ ). Arguing as above, we obtain $c_{01} \equiv c_{11} \equiv$ $c_{21} \equiv 0$. Finally, the system admits only trivial solutions. The second statement of the lemma is proved.

Now let us prove the last statement of the lemma. Our first goal is to prove that if $\alpha_{i} \neq 0$, then the components $c_{0 i}, c_{1 i}, c_{2 i}$ of the solution are identically zero. Indeed, otherwise, arguing as in the proof of the second statement, 
we come to the system $M_{1} c_{1}=0$, where $c_{1}$ is the column with components $c_{03}, c_{13}, c_{23}$ and $M_{1}$ is the 3-by-3 matrix from the proof of the second proposition.

If $\alpha_{3} \neq 0$ then the determinant of the matrix is still nonzero at almost every point (because the term at $e^{-2 x}$ is equal to $5 \alpha_{2}{ }^{4} K(B-2)$ ), and $B \neq 2$ by assumption). Then, the components $c_{03}, c_{13}, c_{23}$ vanish. Doing the same for every $i$, we obtain that if $\alpha_{i} \neq 0$ then $c_{0 i} \equiv c_{1 i} \equiv c_{2 i} \equiv 0$.

Finally, without loss of generality, we can assume that $\alpha_{1}=\alpha_{2}=\alpha_{3}=0$, so our system becomes:

$$
\begin{array}{r}
\frac{\mathrm{d}}{\mathrm{dx}}\left(\begin{array}{c}
c_{01} \\
c_{02} \\
c_{03} \\
c_{11} \\
c_{12} \\
c_{13} \\
c_{22} \\
c_{22} \\
c_{23}
\end{array}\right)=\left(\begin{array}{ccccccccc}
\frac{2}{3} B & 0 & 0 & 0 & 0 & 0 & 0 & 0 & 0 \\
0 & \frac{2}{3} B & 0 & 0 & 0 & 0 & 0 & 0 & 0 \\
0 & 0 & \frac{2}{3} B & 0 & 0 & 0 & 0 & 0 & 0 \\
0 & -1 & 0 & -\frac{1}{3} B & 0 & 0 & 0 & 0 & 0 \\
0 & 0 & -2 & 0 & -\frac{1}{3} B & 0 & 0 & 0 & 0 \\
0 & 0 & 0 & 0 & 0 & -\frac{1}{3} B & 0 & 0 & 0 \\
2 D e^{-2 x} & 0 & 0 & 0 & -1 & 0 & -\frac{4}{3} B & 0 & 0 \\
0 & 2 D e^{-2 x} & 0 & 0 & 0 & -2 & 0 & -\frac{4}{3} B & 0 \\
0 & 0 & 2 D e^{-2 x} & 0 & 0 & 0 & 0 & 0 & -\frac{4}{3} B
\end{array}\right)\left(\begin{array}{l}
c_{01} \\
c_{02} \\
c_{03} \\
c_{11} \\
c_{12} \\
c_{21} \\
c_{22} \\
c_{23}
\end{array}\right) \\
\left(\begin{array}{ccc}
0 & -1 & 0 \\
0 & 0 & -2 \\
0 & 0 & 0
\end{array}\right)\left(\begin{array}{l}
c_{21} \\
c_{22} \\
c_{23}
\end{array}\right)=D e^{-2 x}\left(\begin{array}{c}
c_{11} \\
c_{12} \\
c_{13}
\end{array}\right),
\end{array}
$$

This system can easily be solved. Indeed, assume first $B \neq 1$. The second and the third equations of (20) give

$$
c_{02}(x)=C_{2} e^{2 / 3 B x}, c_{03}(x)=C_{1} e^{2 / 3 B x} .
$$

The last equation of (21) gives $c_{13}=0$. Substituting all these into the two last equations of (20) gives two ordinary differential equations

$$
\left.\begin{array}{l}
\frac{\mathrm{d}}{\mathrm{dx}} c_{12}(x)+4 / 3 B c_{12}(x)=2 D C_{2} e^{(2 / 3 B-2) x} \\
\frac{\mathrm{d}}{\mathrm{dx}} c_{13}(x)+4 / 3 B c_{13}(x)=2 D C_{1} e^{(2 / 3 B-2) x}
\end{array}\right\}
$$

whose solutions are

$$
\begin{aligned}
& c_{12}(x)=e^{-4 / 3 B x} C_{4}+\frac{e^{-2 x+2 / 3 B x} D C_{2}}{-1+B} \\
& c_{23}(x)=e^{-4 / 3 B x} C_{3}+\frac{e^{-2 x+2 / 3 B x} D C_{1}}{-1+B} .
\end{aligned}
$$

Substituting all these into the fourth and fifth equation of (20), we obtain

$$
\left.\begin{array}{cl}
(B-1)(B-2) C_{4}\left(e^{(-4 / 3 B+2) x}+1\right) & =D C_{2}(2 B-1) e^{2 / 3 x(B-1)} \\
(B-1)(B-2) C_{3} e^{-2 x(B-1)} & =D C_{1}(2 B-1)
\end{array}\right\}
$$

implying $C_{1}=C_{2}=C_{3}=C_{4}=0$. Therefore, $c_{02} \equiv c_{03} \equiv c_{12} \equiv c_{13} \equiv 0$, which, in view of equations (21), implies $c_{22} \equiv c_{23} \equiv 0$. 
Finally, $c_{02} \equiv c_{03} \equiv c_{12} \equiv c_{13} \equiv c_{22} \equiv c_{23} \equiv 0$, and the equations(20) become

$$
\frac{\mathrm{d}}{\mathrm{dx}}\left(\begin{array}{l}
c_{01} \\
c_{03}
\end{array}\right)=\left(\begin{array}{cc}
2 / 3 B & 0 \\
2 D e^{-2 x} & -4 / 3 B
\end{array}\right)\left(\begin{array}{l}
c_{01} \\
c_{03}
\end{array}\right)
$$

implying $c_{01}=$ Const $_{1} e^{2 / 3 B x}, c_{03}=$ Const $_{1}\left(\frac{D}{B-1} e^{2(B-1) x}+H\right) e^{-4 / 3 B x}$ which means that the matrix $a$ is as in Lemma 6 .

The case $B=1$ is completely similar. In this case instead of (24) and (25) we have:

$$
c_{12}(x)=\left(2 D C_{2} x+C_{4}\right) e^{-4 / 3 x}, c_{13}(x)=\left(2 D C_{1} x+C_{3}\right) e^{-4 / 3 x} .
$$

Substituting in the fifth and sixth equation of (20) we obtain:

$$
\left.\begin{array}{rl}
2 D C_{2} e^{-4 / 3 x}-2 D C_{2} e^{-4 / 3 x} x-C_{4} e^{-4 / 3 x}+2 C_{1} e^{2 / 3 x} & =0 \\
\frac{4}{3}\left(2 D C_{1} x+C_{3}\right) e^{-4 / 3 x} & =0
\end{array}\right\}
$$

implying $C_{1}=C_{2}=C_{3}=C_{4}=0$. Therefore, $c_{02} \equiv c_{03} \equiv c_{12} \equiv c_{13} \equiv 0$, which, in view of equations (21), implies $c_{22} \equiv c_{23} \equiv 0$. Therefore the equations (201) become

$$
\frac{\mathrm{d}}{\mathrm{dx}}\left(\begin{array}{c}
c_{01} \\
c_{03}
\end{array}\right)=\left(\begin{array}{cc}
2 / 3 & 0 \\
2 D e^{-2 x} & -4 / 3
\end{array}\right)\left(\begin{array}{c}
c_{01} \\
c_{03}
\end{array}\right),
$$

implying $c_{01}=$ Const $_{1} e^{2 / 3 x}, c_{03}=$ Const $_{1}(2 D x+H) e^{-4 / 3 x}$, which means that the matrix $a$ is as in Lemma 6. Lemma 6 is proved.

\subsection{Metrics admitting precisely three vector fields}

In the previous section we obtained a list of all the metrics with $\operatorname{dim}(\mathfrak{p})=2$. The goal of this section is to obtain the list of the metrics with $\operatorname{dim}(\mathfrak{p})=2$. Because of Lemmas 3 and 4, without loss of generality, we can and will assume that our connection is

$$
y^{\prime \prime}(x)=\frac{1}{2} y^{\prime}(x)+D e^{-2 x}\left(y^{\prime}(x)\right)^{3}, \quad \text { where } D \neq 0,
$$

and that every projective vector field is a linear combination of the vector fields

$$
X:=(0,1), Y:=(1, y), Z:=\left(2 y, 1+y^{2}\right) .
$$

Let us show that a metric having precisely three projective vector fields has a Killing vector field. Because of Corollary 1, it is sufficient to find a metric whose projective connection is (28) and that admits a Killing vector field. A metric satisfying both properties is

$$
e^{3 x} d x^{2}-2 D e^{x} d y^{2} .
$$

It admits a Killing vector field because its entries are independent of $y$ and its projective connection is (28). Since only the metrics of constant curvature admit more than one Killing vector field, the Killing vector field is unique up 
to multiplication by a constant. As we recalled in Section 2.2.2, the algebra of the projective vector fields is isomorphic to $\mathfrak{s l}(2, \mathbb{R})$. Recall that the algebra $\mathfrak{s l}(2, \mathbb{R})$ is the algebra of the 2 -by- 2 matrices with trace 0 . It is well-known that up to an automorphism of this algebra every element is proportional to one of the following matrixes:

$$
\mathbf{X}:=\left(\begin{array}{cc}
0 & -1 \\
0 & 0
\end{array}\right), \quad \mathbf{Y}:=\left(\begin{array}{cc}
-1 / 2 & 0 \\
0 & 1 / 2
\end{array}\right) \quad \text { or } \quad \mathbf{Z}:=\left(\begin{array}{cc}
0 & -1 \\
1 & 0
\end{array}\right)
$$

Comparing the commutation relations of the vector fields $X, Y, Z$ with the commutative relation of the matrixes $\mathbf{X}, \mathbf{Y}, \mathbf{Z}$ we see that there exists an isomorphism of the algebras that sends the vector field $X, Y, Z$ to the matrixes $\mathbf{X}, \mathbf{Y}, \mathbf{Z}$, respectively.

Thus, without loss of generality we may assume that the Killing vector field is $X, Y$, or $Z$. In the next three sections, we will consider each of these cases in turn.

\subsubsection{Assume $X=(0,1)$ is a Killing vector field.}

Then the components of the matrix $a=g / \operatorname{det}(g)^{2 / 3}$ do not depend on the $y$-coordinate, and the equations (10) read

$$
\begin{aligned}
& \left.\begin{array}{rl}
\frac{\partial a_{11}}{\partial x}-1 / 3 a_{11} & =0 \\
2 \frac{\partial a_{12}}{\partial x}+1 / 3 a_{12} & =0
\end{array}\right\}
\end{aligned}
$$

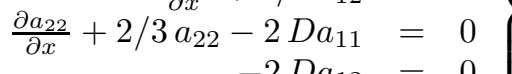

$$
\begin{aligned}
& -2 D a_{12}=0
\end{aligned}
$$

This system can be solved, the solution is

$$
a_{12}=0, \quad a_{11}=e^{1 / 3 x} D^{-2 / 3} 2^{-2 / 3} C_{2}, \quad a_{22}=\left(C_{1} e^{x}+C_{2}\right) 2^{1 / 3} D^{1 / 3} e^{-5 / 3} .
$$

Thus, the metric is

$$
g=\left(\begin{array}{cc}
\frac{e^{3 x}}{C_{2}\left(C_{1} e^{x}+C_{2}\right)^{2}} & 0 \\
0 & \frac{2 D e^{x}}{C_{2}^{2}\left(C_{1} e^{x}+C_{2}\right)}
\end{array}\right)
$$

We see that if $C_{1}=0$, then, after an appropriate scaling $y_{\text {new }}=c y$, the metric coincides with the metric (2a) from Theorem 1 If $C_{1} \neq 0$, then, after an appropriate coordinate change $\left(x_{\text {new }}, y_{\text {new }}\right)=\left(x+c_{1}, c_{2} y\right)$, the metric coincides with the metric (2b) from Theorem 1.

\subsubsection{Assume $Y=(1, y)$ is a Killing vector field.}

Without loss of generality we can assume that $D= \pm 1$. Indeed, $D \neq 0$ by Lemma 4, and, after the scaling $y_{\text {new }}=\alpha y_{\text {old }}$, the projective connection (28) becomes

$$
y^{\prime \prime}=\frac{1}{2} y^{\prime}+\frac{D}{\alpha^{2}}\left(y^{\prime}\right)^{3} .
$$


Consider the coordinate change $\left(x_{\text {new }}, y_{\text {new }}\right)=\left(\left|y_{\text {old }}\right| / e^{x_{\text {old }}}, \ln \left(\left|y_{\text {old }}\right|\right)\right)$. In this new coordinate system, the vector field $Y$ is $(0,1)$ and the projective connection is

$$
y^{\prime \prime}=-\frac{3}{2 x} y^{\prime}+\left(\frac{x}{2}-D x^{3}\right)\left(y^{\prime}\right)^{3} .
$$

Then the components of the matrix $a=g / \operatorname{det}(g)^{2 / 3}$ do not depend on the $y$-coordinate, and the equations (10) have the form

$$
\left.\begin{array}{rl}
\frac{\partial a_{11}}{\partial x}+\frac{a_{11}}{x} & =0 \\
2 \frac{\partial a_{12}}{\partial x}-\frac{a_{12}}{x} & =0 \\
\frac{\partial a_{22}}{\partial x}-2 \frac{a_{22}}{x}-\left(x-2 D x^{3}\right) a_{11} & =0 \\
-\left(x-2 D x^{3}\right) a_{12} & =0
\end{array}\right\}
$$

This system can be solved, the solution $a$ and the metric $g=a / \operatorname{det}(a)^{2}$ are

$$
\begin{gathered}
a=\left(\begin{array}{cc}
\frac{C_{2}}{x} & 0 \\
0 & x\left(C_{1} x-C_{2}\left(1+2 D x^{2}\right)\right)
\end{array}\right) \\
g=\left(\begin{array}{cc}
\frac{1}{C_{2}\left(C_{1} x-C_{2}\left(1+2 D x^{2}\right)\right)^{2} x} & 0 \\
0 & \frac{x}{C_{2}^{2}\left(C_{1} x-C_{2}\left(1+2 D x^{2}\right)\right)}
\end{array}\right)
\end{gathered}
$$

We see that the metric (33) is the metric (2C) with $\varepsilon_{1} \varepsilon_{2}=-1$ from Theorem 1, possible after the coordinate change $x_{\text {new }}=-x$.

\subsubsection{Assume $Z=\left(2 y, 1+y^{2}\right)$ is a Killing vector field.}

As in the previous section, without loss of generality, we can assume $D= \pm 1$.

Consider the coordinate change

$$
x_{\text {new }}=2 \frac{y_{\text {old }}^{2}+1}{e^{x_{\text {old }}}}, \quad y_{\text {new }}=2 \arctan \left(y_{\text {old }}\right)
$$

In this new coordinate system, the vector field $Z$ is $(0,1)$ and the projective connection is

$$
y_{x x}=-\frac{3}{2 x} y_{x}-\left(\frac{x}{2}+D x^{3}\right) y_{x}^{3} .
$$

Then the components of the matrix $a=g / \operatorname{det}(g)^{2 / 3}$ do not depend on the $y$-coordinate, and the equations (10) are

$$
\left.\begin{array}{rl}
\frac{\partial a_{11}}{\partial x}+\frac{a_{11}}{x} & =0 \\
2 \frac{\partial a_{12}}{\partial x}-\frac{a_{12}}{x} & =0 \\
\frac{\partial a_{22}}{\partial x}-2 \frac{a_{22}}{x}-\left(x+2 D x^{3}\right) a_{11} & =0 \\
-\left(x+2 D x^{3}\right) a_{12} & =0
\end{array}\right\}
$$

This system can be solved. The solution $a$ and the metric $g=a / \operatorname{det}(a)^{2}$ are

$$
a=\left(\begin{array}{cc}
\frac{C_{2}}{x} & 0 \\
0 & x\left(C_{1} x+C_{2}\left(1-2 D x^{2}\right)\right)
\end{array}\right),
$$




$$
g=\left(\begin{array}{cc}
\frac{1}{C_{2}\left(C_{1} x+C_{2}\left(1-2 D x^{2}\right)\right)^{2} x} & 0 \\
0 & \frac{x}{C_{2}^{2}\left(C_{1} x+C_{2}\left(1-2 D x^{2}\right)\right)}
\end{array}\right) .
$$

We see that, the metric (35) is the metric (2c) with $\varepsilon_{1} \varepsilon_{2}=1$ from Theorem1 possible after the coordinate change $x_{\text {new }}=-x$.

\subsection{Why are the metrics from Theorem 1 mutually differ- ent?}

Every metric from Theorem 1 has its own individual properties invariant with respect to local isometries that distinguish it within the metrics from Theorem 1.

The individual properties we will use are

- the structure of the algebra of the projective vector fields and the place of the Killing vector field in this algebra.

- The following three functions:

- Scalar curvature $R:=\sum_{i, j, k} R_{i j k}^{i} g^{j k}$

- The square of the length of the derivative of the scalar curvature $I:=\sum_{i, j} g^{i j} \frac{\partial R}{\partial x_{i}} \frac{\partial R}{\partial x_{j}}$

- The laplacian of the scalar curvature

$$
\Delta_{g} R:=\frac{1}{\sqrt{\operatorname{det}(g)}} \sum_{i, j} \frac{\partial}{\partial x_{i}}\left(g^{i j} \sqrt{\operatorname{det}(g)} \frac{\partial R}{\partial x_{j}}\right)
$$

It is easy to distinguish the metrics (1a), (1b), and (1c) from the metrics (2a), (2b), and (2c). Indeed, the metrics (1a), (1b), and (1c) have a two-dimensional space of projective vector fields while the metrics (2a), (2b), and (2C) each have a three-dimensional space of projective vector fields.

Let us distinguish the metrics (2a), (2b), and (2c). By construction, the Killing vector field for the metrics (2a), (2b) (for the metric (2c), respectively), is the element of the Lie algebra of projective vector fields that is isomorphic to $\mathfrak{s l}(2, \mathbb{R})$, is conjugate to $\mathbf{X}$ from (31) ( $\mathbf{Y}$ or $\mathbf{Z}$, respectively). Thus, the metrics (2a), (2b) cannot be isometric to (2c).

Let us distinguish the metrics (2a) and (2b). By direct calculation, one can

see that $\frac{I}{9 R^{3}}$ is equal to 1 for the metric (2a) and to $\frac{8\left(e^{x}+\varepsilon_{2}\right)^{4}}{\varepsilon_{2}\left(3 e^{x}+2 \varepsilon_{2}\right)^{3}}$ for the metric (2b). Thus, the metrics (2a) and (2b) are different.

It is easy to see that two metrics (2a) corresponding to different values of the parameters $\varepsilon_{i}$ are not mutually isometric. Indeed, the $\varepsilon_{i}$ are determined by the signature of the metric and by the sign of the square of the length of the Killing vector field.

Now let us show that the metrics (2C) corresponding to the different values of the parameters $a, c$, and $\varepsilon_{i}$ are different. 
By direct calculation, we see that for the metrics (2c) the functions of $R, I$, $\Delta_{g} R$ are as follows

$$
\begin{aligned}
R & =\left(3 c x^{2}+4 x^{3}+6 \varepsilon_{2} x+1 / 2 \varepsilon_{2} c\right) / a \\
I & =\left(c x+2 x^{2}+\varepsilon_{2}\right)^{4} x / a^{3} \\
\Delta_{g} R & =\left(2 \varepsilon_{2}+5 c x+16 x^{2}\right)\left(c x+2 x^{2}+\varepsilon_{2}\right)^{2} / a^{2} .
\end{aligned}
$$

We see that the mapping

$$
\left(R, I, \Delta_{g} R\right): \mathbb{R} \backslash\left\{x \in \mathbb{R}:\left(c x+2 x^{2}+\varepsilon_{2}\right) x=0\right\} \rightarrow \mathbb{R}^{3}
$$

is an analytic curve in $\mathbb{R}^{3}$, and can be completed at $\left\{x \in \mathbb{R}:\left(c x+2 x^{2}+\varepsilon_{2}\right) x=\right.$ $0\}$. If metrics (2c) corresponding to different values of the parameters $a, c, \varepsilon_{2}$ are isometric, the images of the corresponding curves coincide (as subsets of $\mathbb{R}^{3}$ ), which is not the case.

Indeed, the point $x=0$ is determined by the the condition $I=0, \Delta_{g} R \neq 0$. At this point $R=\varepsilon_{2} \frac{c}{2 a}$ and $\Delta_{g} R=2 \varepsilon_{2}^{3} / a^{2}$. Since $a>0$ and $\varepsilon_{2}= \pm 1$, the curves corresponding to different values of the parameters are different, and therefore the metrics (2C) corresponding to different values of the parameters $a, c$ and $\varepsilon_{2}$ are different as well.

The remaining parameter $\varepsilon_{1}$ determines the role of the Killing vector field in the Lie algebra of projective vector fields: if $\varepsilon_{1} \varepsilon_{2}=-1\left(\varepsilon_{1} \varepsilon_{2}=1\right.$, respectively), then the Killing vector field corresponds to the matrix $\mathbf{Y}$ (Z, respectively) from (31).

Now let us distinguish the metrics (1a), (1b), (1c), and (2b) corresponding to different values of the parameters. In order to do this, let us observe that any isometry between any two of these metrics must send $x$ to $x+x_{0}$. Indeed, the vector $(1,0)$ can be canonically given in isometry-invariant terms as follows: For every Killing vector field $K$, consider the projective vector field $v$ such that

$$
[K, v]=K \text {. }
$$

It is easy to see two such vector fields $u, v$ satisfy $u-v=\lambda K$. Indeed, every Killing vector field for any of these metrics has the form $\alpha(0,1)$, and every projective vector field $v$ with the property 36 has the form $\beta(0,1)+\gamma(1, y)$. (In the cases (1a), (1b), (1c) this is because the space of projective vector fields is two-dimensional. In the case (2b), this is because in $\mathfrak{s l}(2, \mathbb{R})$ the relations $[K, v]=K$ and $[K, u]=K$ imply that $K, u, v$ are linearly dependent.) Then, the relation $[K, v]=K$ implies $\gamma=1$, i.e., such vector field $v$ is uniquely defined up to addition of $\lambda(0,1)$

Thus, the projective vector field satisfying the condition (36) must have $\gamma=1$, and its projection to the normal distribution to the Killing vector field is $(1,0)$. Thus, an isometry between any two of the metrics (1a), (1b), (1c) must send $x$ to $x+x_{0}$.

By direct calculation, we obtain that the function $R$ for the metrics (1a), 
(1b), (1c), and (2b) takes the forms

$$
\begin{aligned}
& R_{\text {年 }}=\varepsilon_{1} b e^{-(b+2) x} \\
& R_{\text {(1b }}=\frac{\varepsilon_{2} b}{2 a}\left((b+2) e^{-2 x}+2 \varepsilon_{2} e^{-(b+2) x}\right) \\
& R_{\text {[1] }}=-\frac{1}{2 a}(2 x+1) e^{-2 x} \\
& R_{\underline{2 \mathrm{~b}}}=\frac{\varepsilon_{2}\left(3 e^{x}+2 \varepsilon_{2}\right)}{2 a e^{3 x}} .
\end{aligned}
$$

Clearly, the change $x_{\text {new }}=x+c$ cannot translate any of these functions to the same functions corresponding to different values of the parameters. The remaining parameter ( $\varepsilon_{2}$ for (1a), $\varepsilon_{1}$ for (1b) and for (2b), $\varepsilon$ for (1c)) is determined uniquely by the sign of the square of the length of the Killing vector.

Thus, all the metrics from Theorem 1 are mutually different. Theorem 1 is proved.

\subsection{Proof of Corollary 3}

We will first prove that if the metrics $g$ and $\bar{g}$ on $M^{2}$ are projectively equivalent, then the spaces $\mathcal{I}(g)$ and $\mathcal{I}(\bar{g})$ are isomorphi 14 . The canonical isomorphism is given by $h \mapsto\left(\frac{\operatorname{det} \bar{g}}{\operatorname{det} g}\right)^{2 / 3} h$.

Indeed, the re-parametrization map

$$
\eta: T M \backslash M \rightarrow T M \backslash M, \quad \eta(\xi)=\frac{|\xi|_{\bar{g}}}{|\xi|_{g}} \xi,
$$

where $T M \backslash M$ denotes the tangent bundle without its zero section, takes the orbits of the geodesic flow of the metric $\bar{g}$ to the orbits of the geodesic flow of the metric $g$. Then, for every $h \in \mathcal{I}(g)$, the function

$$
\eta^{*} h: T M \rightarrow \mathbb{R}, \quad \eta^{*} h(\xi)=\left(\frac{|\xi|_{\bar{g}}}{|\xi|_{g}}\right)^{2} h(\xi)=\frac{\bar{g}(\xi, \xi)}{g(\xi, \xi)} h(\xi)
$$

is constant on the orbits of the geodesic flow of $\bar{g}$, i.e., is an integral of the geodesic flow of $\bar{g}$. Using that the functions $\bar{E}(\xi):=\bar{g}(\xi, \xi)$ and $\bar{I}(\xi):=$ $g(\xi, \xi)\left(\frac{\operatorname{det}(\bar{g})}{\operatorname{det}(g)}\right)^{2 / 3}$ are integrals of the geodesic flow of $\bar{g}$, we obtain that the function $\left(\frac{\operatorname{det}(\bar{g})}{\operatorname{det}(g)}\right)^{2 / 3} h=\frac{\bar{I}}{E} \eta^{*} h$ is an integral of the geodesic flow of $\bar{g}$ as well, i.e., the linear mapping $h \mapsto\left(\frac{\operatorname{det}(\bar{g})}{\operatorname{det}(g)}\right)^{2 / 3} h$ maps $\mathcal{I}(g)$ to $\mathcal{I}(\bar{g})$. Since we obtain the inverse mapping by interchanging $g$ and $\bar{g}$, the mapping $h \mapsto\left(\frac{\operatorname{det} \bar{g}}{\operatorname{det} g}\right)^{2 / 3} h$ is an isomorphism.

\footnotetext{
${ }^{14}$ This statement reflects the fact that the Killing equations are projectively invariant, see 44 7 .
} 
A point $p \in M^{2}$ will be called regular, if two vectors from $\mathfrak{p}(g)$ are linearly independent at $p$. As we explained in Sections 2.2.2 2.2.3 the set of regular points is open and everywhere dense.

Let us first prove Corollary 3 in a small neighborhood of a regular point. From Lemma 4 it follows, that if $\mathfrak{p}(g)=3$, then the projective connection has the form (28) in a certain local coordinate system near every regular point. Since the projective connection of the metric (30) is (28), it is sufficient to show that the metric (30) is superintegrable, which is indeed the case since the functions

$$
\begin{aligned}
H & =\frac{1}{2} e^{3 x} d x^{2}-D e^{x} d y^{2} \\
F_{1} & =e^{2 x} d y^{2} \\
F_{2} & =y H+e^{3 x} d x d y \\
F_{3} & =y F_{2}+2 e^{3 x}\left(y d x d y+4 D^{2} d y^{2}\right)
\end{aligned}
$$

are linearly independent integrals of the geodesic flow of the metric (30). Thus, the restriction of every metric of nonconstant curvature with $\operatorname{dim}(\mathfrak{p}(g))=3$ to a neighborhood of a regular point is superintegrable.

Let us now prove Corollary 3 at every point. We will use the following observation from [24, 29]:

If $Z \in \mathfrak{p}(g)$, then the function $I_{Z}: T M^{2} \rightarrow \mathbb{R}$ defined by

$$
I_{Z}(\xi)=-\left(\mathcal{L}_{Z} g\right)(\xi, \xi)+\frac{2}{3} \operatorname{trace}\left(g^{-1} \mathcal{L}_{Z} g\right) g(\xi, \xi)
$$

lies in $\mathcal{I}(g)$.

This observation implies that if $Z \in \mathfrak{p}(g)$ is a Killing vector field on a certain open set, then it is a Killing vector field everywhere. Indeed, if $I_{Z} \in \mathcal{I}(g)$ vanishes on the open subset, it vanishes everywhere, since it is constant on the orbits on the geodesic flow.

We have shown that $\operatorname{dim}\left(\mathcal{I}\left(g_{\mid U}\right)\right)=4$ for a certain neighborhood $U$ of a regular point. By the result of Koenigs we recalled in Section 2.2.4, in this neighborhood there exists a Killing vector field. As we explained above, this implies the existence of a Killing vector field $K \in \mathfrak{p}(g)$ defined on the whole $M^{2}$.

Consider the linear mapping $F \mapsto Z_{F}$ from the proof of Lemma 2 and the integral $F_{K}$ from Remark 5 . Since $\operatorname{span}\left(F_{K}\right)$ is the kernel of this mapping, the mapping induces an isomorphism between $\mathfrak{p}(g)$ and the quotient space $\mathcal{I}\left(g_{\mid U}\right)_{/ \operatorname{span}\left(F_{K}\right)}$. If two such neighborhoods $U_{1}$ and $U_{2}$ intersect, then the constructed isomorphisms coincide on the intersection and, therefore, induce an isomorphism between $\mathfrak{p}(g)$ and $\mathcal{I}\left(g_{\mid U_{1} \cup U_{2}}\right) / \operatorname{span}\left(F_{K}\right)$. Thus, for every connected component $U_{\text {Reg }}$ of the set of the regular points, $\mathfrak{p}(g)$ and $\mathcal{I}\left(g_{\mid U_{\mathrm{Reg}}}\right) / \operatorname{span}\left(F_{K}\right)$ are isomorphic.

The set of singular (= not regular) points is obviously invariant w.r.t. Killing vector field. Let us show that the Killing vector field vanishes at singular points.

Indeed, by Theorem 1 the universal cover of $U_{R e g}$ is isometric to a connected domain of $\mathbb{R}^{2}$ with the metric $(2 \mathrm{a}),(2 \mathrm{~b})$, or $(2 \mathrm{c})$. We denote the isometry by $\phi$. If $p \in M^{n}$ approaches (the lift of) a singular point, the $x$-coordiate of the 
point $\phi(p)$ tends to $\pm \infty$, or to a value such that the metric is not defined, i.e., to the roots of the equation $e^{x}+\varepsilon_{2}=0$ for the metric $(2 \mathrm{~b})$ and of the equation $\left(c x+x^{2}+\varepsilon_{2}\right) x=0$ for the metric (2c). It is easy to check that in all these cases the scalar curvature $R$ converges to infinity, or the length of every Killing vector field converges to infinity, or the length of every Killing vector field converges to zero. (The formulae for the scalar curvature are in the previous section). Thus, the Killing vector field vanishes at singular points.

Then, the set of singular points consist of a collection of isolated points, so that the set of the regular points has only one connected component $U_{\mathrm{Reg}}$. As we explained above, $\mathfrak{p}(g)$ and $\mathcal{I}\left(g_{\mid U_{\operatorname{Reg}}}\right) / \operatorname{span}\left(F_{K}\right)$ are isomorphic implying that $g_{\mid U_{\mathrm{Reg}}}$ is superintegrable. Since the integrals are preserved by the geodesic flow, the metric $g$ is superintegrable on the whole manifold. Indeed, for every $I \in \mathcal{I}\left(g_{\mid U_{\text {Reg }}}\right)$, for every singular point $p$ and for every geodesic $\gamma$ such that $\gamma(0)=p$ and $\dot{\gamma}(0) \neq 0$, we put $I(\dot{\gamma}(0))=I(\dot{\gamma}(\varepsilon))$, where $1>>|\varepsilon| \neq 0$. The obtained function is evidently smooth, quadratic in velocities and constant on the orbits of the geodesic flow. Corollary 3 is proved.

\section{References}

[1] A. V. Aminova, A Lie problem, projective groups of two-dimensional Riemann surfaces, and solitons, Izv. Vyssh. Uchebn. Zaved. Mat. 1990, no. 6, 3-10; translation in Soviet Math. (Iz. VUZ) 34 (1990), no. 6, 1-9.

[2] A. V. Aminova, Projective transformations of pseudo-Riemannian manifolds. Geometry, 9. J. Math. Sci. (N. Y.) 113 (2003), no. 3, 367-470.

[3] E. Beltrami, Resoluzione del problema: riportari i punti di una superficie sopra un piano in modo che le linee geodetische vengano rappresentante da linee rette, Ann. Mat., 1 (1865), no. 7, 185-204.

[4] S. Benenti, Special symmetric two-tensors, equivalent dynamical systems, cofactor and bi-cofactor systems, Acta Appl. Math. 87(2005), no. 1-3, 3391.

[5] E. Cartan, Sur les variétés à connexion projective, Bull. Soc. Math. France 52 (1924), 205-241.

[6] G. Darboux, Leçons sur la théorie générale des surfaces, Vol. III, Chelsea Publishing, 1896.

[7] M. Eastwood, Notes on projective differential geometry, Symmetries and Overdetermined Systems of Partial Differential Equations (Minneapolis, MN, 2006), 41-61, IMA Vol. Math. Appl., 144(2007), Springer, New York.

[8] M. Eastwood, V. S. Matveev, Metric connections in projective differential geometry, Symmetries and Overdetermined Systems of Partial Differential Equations (Minneapolis, MN, 2006), 339-351, IMA Vol. Math. Appl., 144(2007), Springer, New York. 
[9] N. Ibragimov, Essay on the group analysis of ordinary differential equations, Znanie, Moscow, 1991.

[10] E.G. Kalnins, J.M. Kress, W. Miller Jr., Second order superintegrable systems in conformally flat spaces. II. The classical two-dimensional Stäckel transform, J. Math. Phys. 46 (2005), no. 5.

[11] M. S. Knebelman, On groups of motion in related spaces, Amer. J. Math., 52 (1930), 280-282.

[12] G. Koenigs, Sur les géodesiques a intégrales quadratiques, Note II from Darboux' 'Leçons sur la théorie générale des surfaces', Vol. IV, Chelsea Publishing, 1896.

[13] O. Kowalski, Z. Vlasek, Classification of locally projectively homogeneous torsion-less affine connections in the plane domains, Beiträge zur Algebra und Geometrie 48(2007), no.1, 11-26.

[14] J.-L. Lagrange, Sur la construction des cartes géographiques, Novéaux Mémoires de l'Académie des Sciences et Bell-Lettres de Berlin, 1779.

[15] S. Lie, Untersuchungen über geodätische Kurven, Math. Ann. 20 (1882); Sophus Lie Gesammelte Abhandlungen, Band 2, erster Teil, 267-374. Teubner, Leipzig, 1935.

[16] S. Lie, Classification und Integration von gewöhnlichen Differentialgleichungen zwischen $x, y$, de eine Gruppe von Transformationen gestatten, III, Norwegian Archives (1883); also appeared in Mathematische Annalen 32 (1888), 213-281.

[17] S. Lie, Vorlesungen über Differentialgleichungen mit bekannten infinitesimalen Transformation, Teubner, Leipzig, 1912.

[18] R. Liouville, Sur les invariants de certaines équations différentielles et sur leurs applications, Journal de l'École Polytechnique 59 (1889), 7-76.

[19] V. S. Matveev, P. J. Topalov, Trajectory equivalence and corresponding integrals, Regular and Chaotic Dynamics, 3 (1998), no. 2, 30-45.

[20] V. S. Matveev, P. J. Topalov, Geodesic equivalence of metrics on surfaces, and their integrability, Dokl. Math. 60 (1999), no.1, 112-114.

[21] V. S. Matveev and P. J. Topalov, Metric with ergodic geodesic flow is completely determined by unparameterized geodesics, ERA-AMS, 6 (2000), 98104.

[22] V. S. Matveev, Die Vermutung von Obata für Dimension 2, Arch. Math. 82 (2004), 273-281.

[23] V. S. Matveev, Solodovnikov's theorem in dimension two, Dokl. Math. 69 (2004), no. 3, 338-341. 
[24] V. S. Matveev, Lichnerowicz-Obata conjecture in dimension two, Comm. Math. Helv. 81(2005) no. 3, 541-570.

[25] V. S. Matveev, Proof of projective Lichnerowicz-Obata conjecture, J. Diff. Geom. (2007), 75(2007), 459-502.

[26] V. S. Matveev, Geometric explanation of Beltrami theorem, Int. J. Geom. Methods Mod. Phys. 3 (2006), no. 3, 623-629.

[27] Yu. R. Romanovskiu,, Calculation of local symmetries of second-order ordinary differential equations by Cartan's equivalence method, Mat. Zametki 60(1996), no. 1, 75-91, 159; English translation in Math. Notes 60(1996), no. $1-2,56-67$.

[28] P. J. Topalov and V. S. Matveev, Geodesic equivalence via integrability, Geometriae Dedicata 96 (2003), 91-115.

[29] P. J. Topalov, Comutative conservation laws for geodesic flows of metrics admitting projective symmetry, Math. Research Letters 9 (2002), 65-72.

[30] A. Tresse, Sur les invariants différentiels des groupes continus de transformations, Acta Mathematica, 18 (1894), 1-88.

[31] A. Tresse, Détermination des invariants ponctuels de léquation différentielle ordinaire du second ordre $y^{\prime \prime}=\omega\left(x, y, y^{\prime}\right)$. Leipzig. 87 S. gr. $8^{\circ}$. (1896).

[32] J. A. Schouten, Erlanger Programm und Übertragungslehre. Neue Gesichtspunkte zur Grundlegung der Geometrie, Rendiconti Palermo 50 (1926), $142-169$.

[33] A. S. Solodovnikov, Projective transformations of Riemannian spaces, Uspehi Mat. Nauk (N.S.) 11 (1956), no. 4(70), 45-116.

[34] A. S. Solodovnikov, Spaces with common geodesics, Trudy Sem. Vektor. Tenzor. Anal. 11 (1961), 43-102.

[35] A. S. Solodovnikov, Geometric description of all possible representations of a Riemannian metric in Levi-Civita form, Trudy Sem. Vektor. Tenzor. Anal. 12 (1963), 131-173.

[36] A. S. Solodovnikov, The group of projective transformations in a complete analytic Riemannian space, Dokl. Akad. Nauk SSSR 186 (1969), 12621265. 